\title{
Natural Dietary Supplement, Carvacrol, Alleviates LPS-Induced Oxidative Stress, Neurodegeneration, and Depressive-Like Behaviors via the Nrf2/HO-I Pathway
}

This article was published in the following Dove Press journal:

Journal of Inflammation Research

\author{
Komal Naeem (1D ${ }^{1,2, *}$ \\ Lina Tariq Al Kury $\mathbb{D}^{3}$ \\ Faiza Nasar ${ }^{1}$ * \\ Abdullah Alattar ${ }^{4}$ \\ Reem Alshaman ${ }^{4}$ \\ Fawad Ali Shah (D) \\ Arif-ullah Khan' \\ Shupeng $\mathrm{Li}^{2}$ \\ 'Department of Pharmacology, Faculty of \\ Pharmaceutical Sciences, Riphah \\ International University, Islamabad, \\ 747424, Pakistan; ${ }^{2}$ State Key Laboratory \\ of Oncogenomics, School of Chemical \\ Biology and Biotechnology, Shenzhen \\ Graduate School, Peking University, \\ Shenzhen, 518000, People's Republic of \\ China; ${ }^{3}$ College of Natural and Health \\ Sciences, Zayed University, Abu Dhabi, \\ 49153, United Arab Emirates; \\ ${ }^{4}$ Department of Pharmacology and \\ Toxicology, Faculty of Pharmacy, \\ University of Tabuk, Tabuk, 7/42I, Saudi \\ Arabia \\ *These authors contributed equally to \\ this work
}

Correspondence: Fawad Ali Shah Department of Pharmacology, Faculty of Pharmaceutical Sciences, Riphah International University, 7th Avenue, Sector G-7/4, Islamabad, 747424, Pakistan Tel +92-5I-2891835-38

Fax +92-5I-289|47|

Email fawad.shah@riphah.edu.pk

Shupeng Li

State Key Laboratory of Oncogenomics,

School of Chemical Biology and

Biotechnology, Shenzhen Graduate

School, Peking University, Shenzhen,

518000, People's Republic of China

Email lisp@pku.edu.cn
Purpose: Major depressive disorder (MDD) is a debilitating human health condition characterized by mood swings and is associated with a high probability of suicide attempts. Several studies have reported a role of neuroinflammation in MMD, yet the efficacy of natural drug substances on neuroinflammation-associated depression has not been wellinvestigated. The present study examined the neuroprotective effects of carvacrol on lipopolysaccharide (LPS)-induced neuroinflammation, depression, and anxiety-like behavior.

Methods: Male Sprague Dawley rats were divided into two experimental cohorts to determine the effects and the effective dose of carvacrol (whether $20 \mathrm{mg} / \mathrm{kg}$ or $50 \mathrm{mg} / \mathrm{kg}$ ), and further demonstrate the mechanism of action of nuclear factor E2-related factor (Nrf2) in depression.

Results: We found marked neuronal alterations in the cortex and hippocampus of LPSintoxicated animals that were associated with higher inflammatory cytokine expression such as cyclooxygenase (COX2), tumor necrosis factor-alpha (TNF- $\alpha$ ), and c-Jun N-terminal kinase (p-JNK). These detrimental effects exacerbated oxidative stress, as documented by a compromised antioxidant system due to high lipid peroxidase (LPO). Carvacrol (20 mg/kg) significantly reverted these changes by positively modulating the antioxidant gene Nrf2, a master regulator of the downstream antioxidant pathway. To further investigate the role of $\mathrm{Nrf}$, an inhibitor of Nrf2 called all-trans retinoic acid (ATRA) was used, which further exacerbated LPS toxicity with a higher oxidative and inflammatory cytokine level. To further support our notion, we performed virtual docking of carvacrol with the Nrf2-Keap1 target and the resultant drug-protein interactions validated the in vivo findings.

Conclusion: Collectively, our findings suggest that carvacrol $(20 \mathrm{mg} / \mathrm{kg})$ could activate the endogenous master antioxidant Nrf2, which further regulates the expression of downstream antioxidants, eventually ameliorating LPS-induced neuroinflammation and neurodegeneration.

Keywords: depression, neuroinflammation, Nrf2 pathway, lipopolysaccharide, carvacrol, neurodegeneration

\section{Introduction}

Major depressive disorder (MDD) is a devastating human health disorder that is characterized by feelings of worthlessness, lack of motivation, altered mood, and recurrent thoughts of suicide, along with a higher prevalence of suicide. ${ }^{1,2}$ Moreover, depression is known to be the second-leading cause of disease burden for developed countries by 2020 as per the World Health Organization. ${ }^{3}$ Although 
extensive research is underway on existing antidepressants that target the serotonin and/or norepinephrine systems, the risk-benefit ratio demands subsequent alternatives. Moreover, the usefulness of existing antidepressants is further compromised due to delayed therapeutic response and limited patient recovery. ${ }^{4}$ Furthermore, the relatively poor understanding of the mechanisms of antidepressant therapy combined with the complicated prognosis of the disease hinders the treatment strategies. Therefore, an indepth understanding of the mechanisms underlying this disease is required to develop novel treatments for depression. $^{5}$

Multiple studies have proposed that oxidative stress and inflammation can exacerbate the severity of $\mathrm{MDD},{ }^{6}$ as a consistently high level of inflammatory cytokines is found in MDD pathophysiology. ${ }^{7}$ The surge in cytokines are directly related to the permeation of leukocytes and granulocytes to the brain from the surrounding vasculature. ${ }^{8}$ Moreover, resident glial cells are rapidly activated and trigger the release of pro-inflammatory cytokines such as interleukin-1 (IL-1 $\beta$ ), tumor necrotic factor-alpha (TNF- $\alpha$ ), and interleukin-6 (IL-6), which clinically compromises the prognosis of $\mathrm{MDD},{ }^{9-11}$ Lipopolysaccharide (LPS), a bacterial endotoxin, is frequently used to induce behavioral deficits and neurodegeneration and thereby used to model depression and anxiety in rodents. ${ }^{12-14}$ The exact mechanism of LPS-induced neurodegeneration is not known, but multiple studies suggest the involvement of Toll-like receptor (TLR)-4, whose activation leads to elevated levels of inflammatory mediators. ${ }^{15}$ The cross-talk of inflammation and oxidative stress significantly perturbs certain antioxidant proteins such as Nrf-2 and heme oxygenase-1 (HO-1). ${ }^{16-19}$

The transcription factor Nrf2 is an integral part of the host cellular defense mechanism against oxidative stress and electrophilic insult. Nrf2 binds to antioxidant response elements (ARE) at promoter sites of genes that encode several antioxidant/phase-II detoxifying enzymes and other relevant stress-responding factors. ${ }^{20}$ Previous studies revealed the involvement of Nrf2-ARE signaling in attenuating inflammation in several pathologies such as stroke and other disorders. ${ }^{21-23}$ Hence, dysregulation of Nrf2 signaling results in increased susceptibility to oxidative stress and inflammatory damage. ${ }^{24,25}$ Moreover, the neuroprotective role of $\mathrm{Nrf} 2$ in various neurodegenerative disease models has been reported. ${ }^{26}$ Previous studies demonstrated that Nrf2 plays a critical role in the regulation of inflammation and oxidative stress, two processes that are linked to the pathophysiology of depression. ${ }^{27,28}$ Therefore, Nrf2 might be considered a potential pharmacological target for the investigation of depressive behaviors.

Natural drug moieties are an attractive source of new drugs, owing to their rich antioxidant potential. Several natural drugs have protective potential against a variety of mediators, including free radicals and inflammatory factors. ${ }^{29}$ Carvacrol (5-isopropyl-2-methyl phenol), a monoterpene phenol, is extracted as an essential oil from the plant family Labiatae, including the genera Thymbra, Origanum, Satureja, and Thymus. ${ }^{30}$ Carvacrol possesses favorable biological activities possibly due to its antioxidant and anti-inflammatory properties. ${ }^{30}$ Previously carvacrol demonstrated neuroprotective effects in ischemic brain injury by attenuating infarction area. ${ }^{31}$ Furthermore, carvacrol also attenuated behavioral deficits and memory impairment in a rat model possibly by enhancing brainderived neurotrophic factor (BDNF) associated with a decreased expression of TLR4 mRNA. ${ }^{32}$ In another study, Melo et al demonstrated that carvacrol can mitigate depression-like symptoms by modulating dopamine levels. ${ }^{33}$ Similarly, carvacrol attenuated ethanol-induced cognitive deficits by modulating MAPK cascade and mitochondrial pathways. ${ }^{34}$ Taking into consideration the pharmacological value of essential oils and the significance of new drug discovery, the current study aimed to investigate whether carvacrol administration can ameliorate LPSinduced behavioral deficits, neurodegeneration, and the neuroinflammatory cascades in an animal model by targeting the Nrf2-signaling pathway. As per our information and literature survey, we for the first time demonstrated that carvacrol mediated anxiolytic and anti-depressant effects by promoting the Nrf2 dependent downregulation of inflammatory cascades.

\section{Materials and Methods Chemicals and Reagents}

Carvacrol (W224502), glutathione (GSH), trichloroacetic acid (TCA),1-Chloro-2,4-dinitrobenzene (CDNP), $\mathrm{N}$-(1-Naphthyl) ethylenediamine dihydrochloride, 5,5'Dithio-bis-(2-nitrobenzoic acid) (DTNB), LPS, and all-trans retinoic acid (ATRA) were purchased from Sigma-Aldrich (St. Louis, MO, USA). Primary antibodies such as mouse monoclonal anti-HO-1 (SC-136960), mouse monoclonal anti-TNF- $\alpha$ (SC-52B83), mouse monoclonal anti-p-NFאB (SC-271908), rabbit polyclonal anti-Nrf2 (SC-722), mouse 
monoclonal anti-caspase-3 (31A1067), mouse monoclonal anti-Bcl-2 (sc-7382), an ABC Elite kit, and 3,3'-diaminobenzidine peroxidase (DAB) were purchased from Santa Cruz Biotechnology USA. The horseradish peroxidase-conjugated secondary antibody was obtained from Abcam UK (ab6789). ELISA kits for p-NF-kB (Cat \# SUB28069) and Nrf2 (cat. no. SU-B30429) were procured from Shanghai Yuchun Biotechnology (China), and HO-1 (cat. No. E-ELR0488) and TNF- $\alpha$ (cat. No. E-EL-R0019) ELISA kits were purchased from Elabscience.

\section{Animals}

Male Sprague Dawley rats weighing 180-200 g were housed three per cage under a 12 hour light/dark cycle with free access to water and food at the animal house of Riphah Institute of Pharmaceutical Sciences (RIPS) under standard laboratory protocols (temperature: $22 \pm 1^{\circ} \mathrm{C}$; humidity: $50 \% \pm 10 \%$ ). All experimental procedures were carried out according to the guidelines of the Institute of Laboratory Animal Resources, Commission on Life Sciences University, National Research Council (1996), approved by the RIPS Ethical Committee (Reference No: REC/RIPS/2017/010).

\section{Experimental Outline}

Two separate cohorts of animals were used in this study. The first cohort included five groups $(\mathrm{n}=10$ animals/ group): 1) saline (10 mL/kg with 5\% DMSO), 2) LPS (1 mg/kg), 3) LPS + CAR 20 (carvacrol; $20 \mathrm{mg} / \mathrm{kg}$ ), 4) LPS + CAR 50 (carvacrol; $50 \mathrm{mg} / \mathrm{kg}$ ), and 5) LPS + FLX (fluoxetine; $5 \mathrm{mg} / \mathrm{kg}$ ). The second cohort included three groups:1) LPS + ATRA, 2) CAR (20 mg/kg) + ATRA + LPS, and 3) FLX + ATRA + LPS ( $n=8$ animals/group). Carvacrol and fluoxetine were dissolved in normal saline containing 5\% DMSO and administered once daily (i.p.) for five consecutive days (Figure 1). On days three and four, the animals received LPS (1 mg/kg, i.p.) $1 \mathrm{~h}$ after the carvacrol/fluoxetine injection. ${ }^{35}$ On day five, after the last carvacrol/fluoxetine injection, animals were analyzed for depressive- and anxiety-like behaviors (ie, despair) by the sucrose splash test (SST), elevated plus maze (EPM) test, light-dark box (LDB) test, and forced swim test (FST). Following the behavioral tests, the animals were terminally anesthetized with sodium pentobarbital $(60 \mathrm{mg} / \mathrm{kg}$, i.p.). Brain tissues were extracted from the prefrontal cortex and hippocampus and were snap-frozen and stored at $-80^{\circ} \mathrm{C}$. ATRA dissolved in normal saline containing $5 \%$ DMSO was administered $30 \mathrm{~min}$ before LPS administration (i.p.). Carvacrol $(20 \mathrm{mg} / \mathrm{kg})$ and fluoxetine were administered as described above.

\section{Behavioral Studies \\ Sucrose Splash Test (SST)}

The SST was performed to evaluate the depressive-like behavior of rats and was conducted as previously reported. ${ }^{36}$ Grooming behavior indicates depressive symptoms measured by time in seconds. The shorter the time of grooming behavior, the higher the level of depression. The test was performed by spraying a $10 \%$ sucrose solution on the dorsal surface of the rodent's body, and grooming behavior was measured in terms of licking, biting, or scratching the fur to clean the solution off. Grooming time was videotaped for $5 \mathrm{~min}$.

\section{Elevated Plus-Maze (EPM) Test}

An EPM test was conducted to measure LPS-mediated anxiety-like behavior. Briefly, the maze equipment consisted of two oppositely facing open arms and two oppositely facing closed arms (OA, $50 \times 10 \mathrm{~cm}$; CA, $50 \times$ $10 \mathrm{~cm}$ ) in the form of a cross-shaped Plexiglas platform with $40 \mathrm{~cm}$ walls and a height of $50 \mathrm{~cm}$ above the floor in a soundproof room with dim light. Each rat was placed at the central point of the Plexiglas platform with its head facing any open arm. The time spent and the number of entries in each arm were recorded for all animals for 5 min. $^{37}$

\section{Light-Dark Box (LDB) Test}

The LDB test was performed to assess LPS-mediated anxiety-like behavior. LDB equipment comprises of a light and a dark compartment separated by a partition containing a small gap. Each animal was placed in the dark compartment of a light-dark box and was set to freely move around the box for $5 \mathrm{~min}$. The total number of entries and time spent in each compartment was videotaped. Olfactory cues were minimized by ensuring thorough cleaning of the light-dark box with alcohol between tests. $^{38}$

\section{Forced Swim Test (FST)}

The rat was placed in a Plexiglas cylinder that was $70 \mathrm{~cm}$ in height and $30 \mathrm{~cm}$ in diameter and filled with water above a $30 \mathrm{~cm}$ height at a temperature of $23 \pm 1^{\circ} \mathrm{C}$ and then videotaped for $7 \mathrm{~min}$. The last $4 \mathrm{~min}$ were randomly assessed at 5 s intervals. ${ }^{35}$ Climbing, swimming, and floating were the predominant behaviors observed during each $5 \mathrm{~s}$ interval. Rats were categorized as immobile when they floated in 


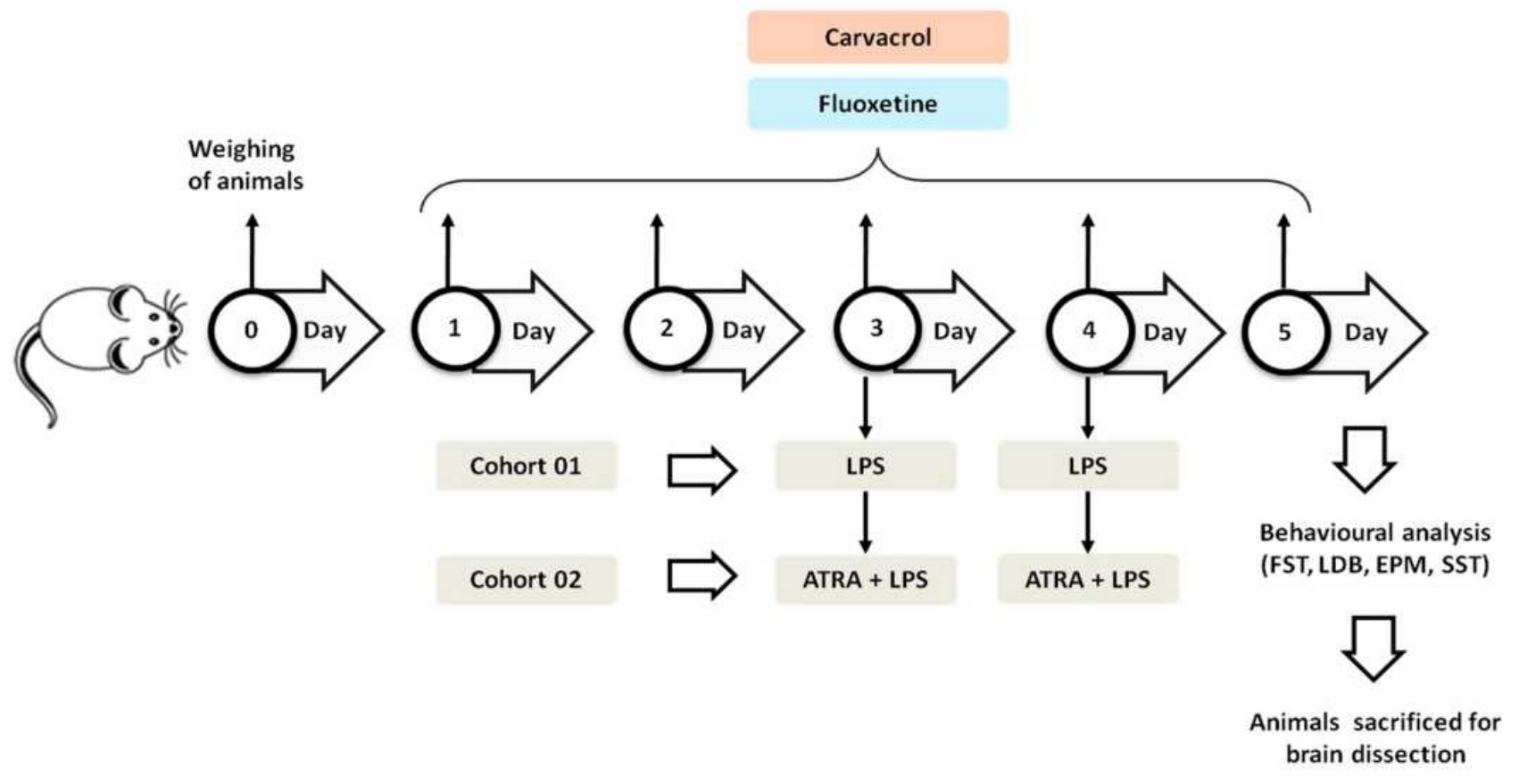

Figure I Experimental outline.

a motionless manner while showing only movements that were necessary to keep their heads above the surface of the water. Horizontal movements were considered swimming and vertical movements were taken as climbing.

\section{Antioxidant Assays}

Determination of Lipid Peroxidation (LPO)

The procedure to determine lipid peroxidation in tissue was followed as previously reported. ${ }^{39}$ Cortical and hippocampal tissues of each rat were homogenized separately in $10 \mathrm{~mL}$ of $20 \mathrm{mM}$ Tris- $\mathrm{HCl}$ at $4{ }^{\circ} \mathrm{C}$ while maintaining $\mathrm{pH}$ at 7.4 in the Polytron homogenizer. The homogenate was then centrifuged at $1000 \mathrm{~g}$ for $10 \mathrm{~min}$ at $4{ }^{\circ} \mathrm{C}$ and the supernatant was collected. A fresh solution of ferric ammonium sulfate was prepared. Forty microliters of the above solution were added to the collected supernatant, which was then incubated at $37{ }^{\circ} \mathrm{C}$ for $30 \mathrm{~min}$. Then, a $0.8 \%$ solution of 2 -thiobarbituric acid (TBA) was prepared by dissolving $400 \mathrm{mg}$ in $50 \mathrm{~mL}$ of water. A total of $75 \mu \mathrm{L}$ of TBA was added to the mixture of the supernatant and ferric ammonium sulfate. Absorbance was measured at a wavelength of $532 \mathrm{~nm}$ using a plate reader.

\section{Reduced Glutathione (GSH) Activity}

The reduced glutathione (GSH) activity was measured with some modification to what has been previously described. ${ }^{40}$ Each well contained $40 \mu \mathrm{L}$ of $0.6 \mathrm{mM}$
DTNB in $0.2 \mathrm{M}$ sodium phosphate while maintaining $\mathrm{pH}$ at 8 , followed by the addition of $6.6 \mu \mathrm{L}$ of sample and $153 \mu \mathrm{L}$ of phosphate buffer to each well. Absorbance was recorded using a plate reader at a wavelength of $412 \mathrm{~nm}$.

\section{Glutathione-S-Transferase (GST) Activity}

GST activity was measured using CDNB as a substrate, as described previously with a slight modification. ${ }^{41}$ Each well was filled with $10 \mu \mathrm{L}$ of $1 \mathrm{mMCDNB}, 10 \mu \mathrm{L}$ of $5 \mathrm{mM}$ reduced glutathione, $270 \mu \mathrm{L}$ of buffer solution, and $10 \mu \mathrm{L}$ of the sample. The absorbance was read at $340 \mathrm{~nm}$ using a plate reader.

\section{Catalase Activity}

Catalase activity was measured by mixing $3 \mathrm{~mL}$ of $\mathrm{H} 2 \mathrm{O} 2$ and $0.05 \mathrm{~mL}$ of tissue supernatant. The absorbance activity was measured at $240 \mathrm{~nm}$ against a blank containing $3 \mathrm{~mL}$ of PBS only. The absorbance is proportional to the $\mathrm{H} 2 \mathrm{O} 2$ level, which is decreased by catalase as it degrades the $\mathrm{H} 2 \mathrm{O} 2$. This is the measure of $\mathrm{H} 2 \mathrm{O} 2$ breakdown and hence is expressed as $\mu \mathrm{mol}$ $\mathrm{H} 2 \mathrm{O} 2$ decomposed per $\mathrm{mg}$ of protein/min. ${ }^{42}$

\section{Histological Preparation}

Rats were decapitated after the behavioral tests, followed by the extraction of brain tissues. Three mm thick sections were cut using a sharp blade and fixed in a $4 \%$ paraformaldehyde solution. The tissues were embedded into paraffin blocks and trimmed to $4 \mu \mathrm{m}$ thin coronal sections using a microtome, and following staining techniques were applied. 


\section{Hematoxylin \& Eosin (H\&E) Staining}

Absolute xylene was used to deparaffinize the tissuecoated slides, followed by rehydration with a gradient ethanol concentration $(100 \%$ to $70 \%)$. Slides were then washed with distilled water and rinsed with the hematoxylin stain for $10 \mathrm{~min}$ to localize the nucleus. The slides were then kept under running water in a glass jar for 10 min and treated with $1 \% \mathrm{HCl}$ and $1 \%$ ammonia water as reported previously. ${ }^{43}$ Eosin solution was used afterward for 5-10 min for cytoplasmic staining. After the specified time, slides were rinsed with water and airdried for a short interval. Graded concentrations (70\%, $95 \%$, and $100 \%$ ) of ethanol were used to rehydrate the slides, which were then cleared with xylene. Finally, the slides were coverslipped using mounting media. A light microscope (Olympus, Japan) was used to capture images of the brain sections. Images were then analyzed using ImageJ software. Five images per slide per group were analyzed with a specific focus on identifying infiltrations, vacuole formation, and neuronal survival. For all groups, images of the same threshold intensity were optimized in the TIFF format.

\section{Immunohistochemical Analysis}

We employed a previously described procedure with slight modifications for immunohistochemical analysis. ${ }^{44}$ After completion of the deparaffinization step, slides were processed by an enzymatic method for antigen retrieval, and then washed with PBS consecutively three times for $5 \mathrm{~min}$. Slides were immersed in $3 \% \mathrm{H} 2 \mathrm{O} 2$ to quench endogenous peroxidase activity followed by washing with PBS. Normal goat serum (5\%) was applied as a blocking serum and slides were incubated for $2 \mathrm{~h}$. Next, the slides were incubated overnight with primary antibodies Bcl2, Caspase 3, TNF- $\alpha$, and $\mathrm{p}-\mathrm{NF} \kappa \mathrm{B}$. The next morning, slides were washed with PBS and incubated for 90 min with the secondary antibody, then incubated with an $\mathrm{ABC}$ kit (Santa Cruz) in a humidified box for $60 \mathrm{~min}$. Slides were then washed with PBS solution and stained with DAB, followed by dehydration with ethanol $(70 \%, 80 \%, 90 \%$, and $100 \%$ ). After dehydration, slides were fixed with xylene and then coverslipped with mounting media. Images were obtained using a light microscope and saved in TIFF format for further quantification by ImageJ software.

\section{ELISA Analysis}

Approximately $70 \mathrm{mg}$ of cortical tissue was homogenized in PBS containing phenylmethylsulfonyl fluoride (PMSF) as a protease inhibitor using a Silent Crusher
M (Heidolph-Germany). The resultant homogenate was then centrifuged at $15,000 \mathrm{rpm}$ at $4^{\circ} \mathrm{C}$ for $20 \mathrm{~min}$, and the supernatant was carefully collected from the top, avoiding the pellet at the bottom. The protein concentration was then determined for each group using a bicinchoninic acid (BCA) kit (Thermo Fisher Scientific, USA) and protein concentration was determined using 96well ELISA plates according to the manufacturer protocols. The readings were taken using an ELISA microplate reader (BioTek ELx808) and the concentration (pg/mL) was then normalized to the total protein content $(\mathrm{pg} / \mathrm{mg}$ total protein).

\section{Western Blot}

All the samples were homogenized using lysis buffer $(1 \mathrm{M}$ Tris- $\mathrm{HCl}, 5 \mathrm{M} \mathrm{NaCl}$, sodium deoxycholate $0.5 \%$, sodium dodecyl sulfate $10 \%$, sodium azide $1 \%$, and NP-40 10\%) further supplemented with a protease inhibitor (PMSF). After centrifugation, the protein concentration of the lysate was determined using a BCA method (Pierce, Rockford, IL, United States) following the guidelines of the manufacturer. An equal amount of protein (30 $\mu \mathrm{g}$ per sample) was electrophoresed on $10 \%$ SDS-PAGE gels, followed by transferring the protein to polyvinylidene fluoride (PVDF) membranes (Millipore, Billerica, MA, United States). After blocking the PVDF membranes using skim milk to reduce binding of the nonspecific protein binding, the PVDF membrane was incubated at $4{ }^{\circ} \mathrm{C}$ for the whole night with the primary antibodies against rat anti-Nrf2, mouse anti-HO-1. $\beta$-actin was used as a loading control. $^{45,46}$ The following day, membranes were incubated with secondary antibodies, and western bands were visualized on $\mathrm{x}$-ray film using ECL detection reagent following the instructions of the manufacturer (Amersham Pharmacia Biotech, Piscataway, NJ, United States).

\section{Molecular Docking}

Molecular docking was performed using the GOLD v5.2.2 (Genetic Optimization of Ligand Docking) package. ${ }^{47}$ The 3D structure of Keap1 in complex with the RA839 inhibitor (PDB ID: 5CGJ) was retrieved from the RCSB protein data bank. ${ }^{48}$ Keap1 was removed from all heteroatoms, water molecules, and other non-desirable entities before initiating the docking simulation, followed by the addition of hydrogen atoms and detection of the Keap1 docking site from the attached inhibitor (RA839). The online database PubChem was used to retrieve the 2D structure of carvacrol, which was then processed for 
docking in DS v4.5 (Discovery Studio). Following the docking protocol, approximately 100 different conformations of carvacrol were created by employing the genetic algorithm (GA) of the GOLD package. Moreover, the scoring function was performed and the results were analyzed using the software ChemPLP (piecewise linear potential). The highest value of ChemPLP determined the best inhibitory confirmation as well as polar and non-polar interactions of carvacrol with the active binding residues of Keap1. Assessment and interpretation of docking results were carried out using DS and GOLD packages.

\section{Statistical Analysis}

The results obtained were expressed as mean \pm standard error of the mean (SEM) and statistically analyzed by applying oneway ANOVA followed by Bonferroni multiple comparisons as post-hoc. A value of $p<0.05$ was considered statistically significant. The bar graphs were analyzed using GraphPad Prism 6.0 (GraphPad, San Diego, CA, USA). The symbol \# shows a significant difference relative to the saline group, and
* shows a significant difference relative to the LPS group, while $\$$ represents a significant difference to ATRA + LPS.

\section{Results}

\section{Effects of Carvacrol on the LPS-Induced Behavioral Deficit}

LPS-treated animals demonstrated a significant decrease in exploratory behavior as shown by the fewer entries to the open arms in the EPM test and by less time spent in the open arms compared to the saline group (Figure 2A and B,

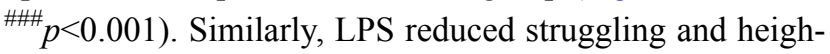
tened immobility in the FST (Figure 2C and D,

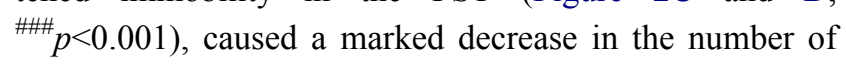
entries and duration in the light compartment in the LDB test (Figure $2 \mathrm{E}$ and $\mathrm{F},{ }^{\#} p<0.001$ ), and significantly reduced grooming time in the SST (Figure 2G,

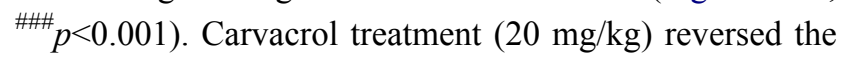
LPS-induced behavioral deficits and was accompanied by a greater number of entries and duration of time spent in the open arms in the EPM test (Figure $2 \mathrm{~A}$ and $\mathrm{B}$,
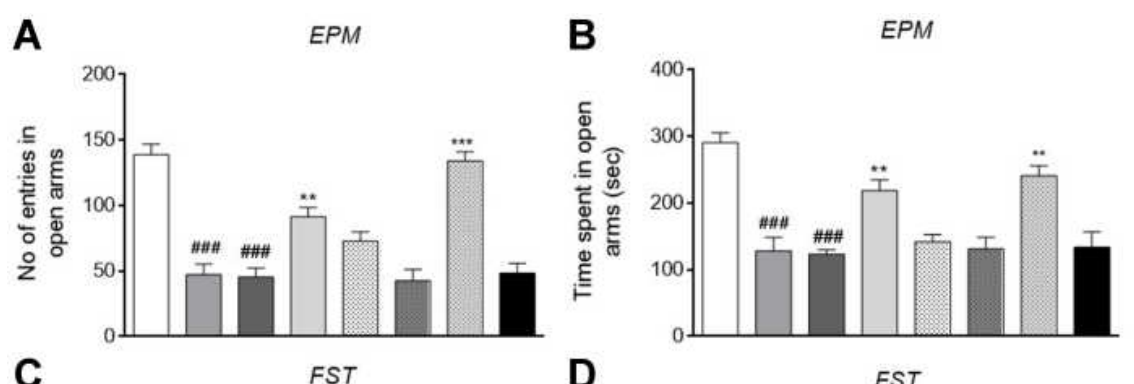

D
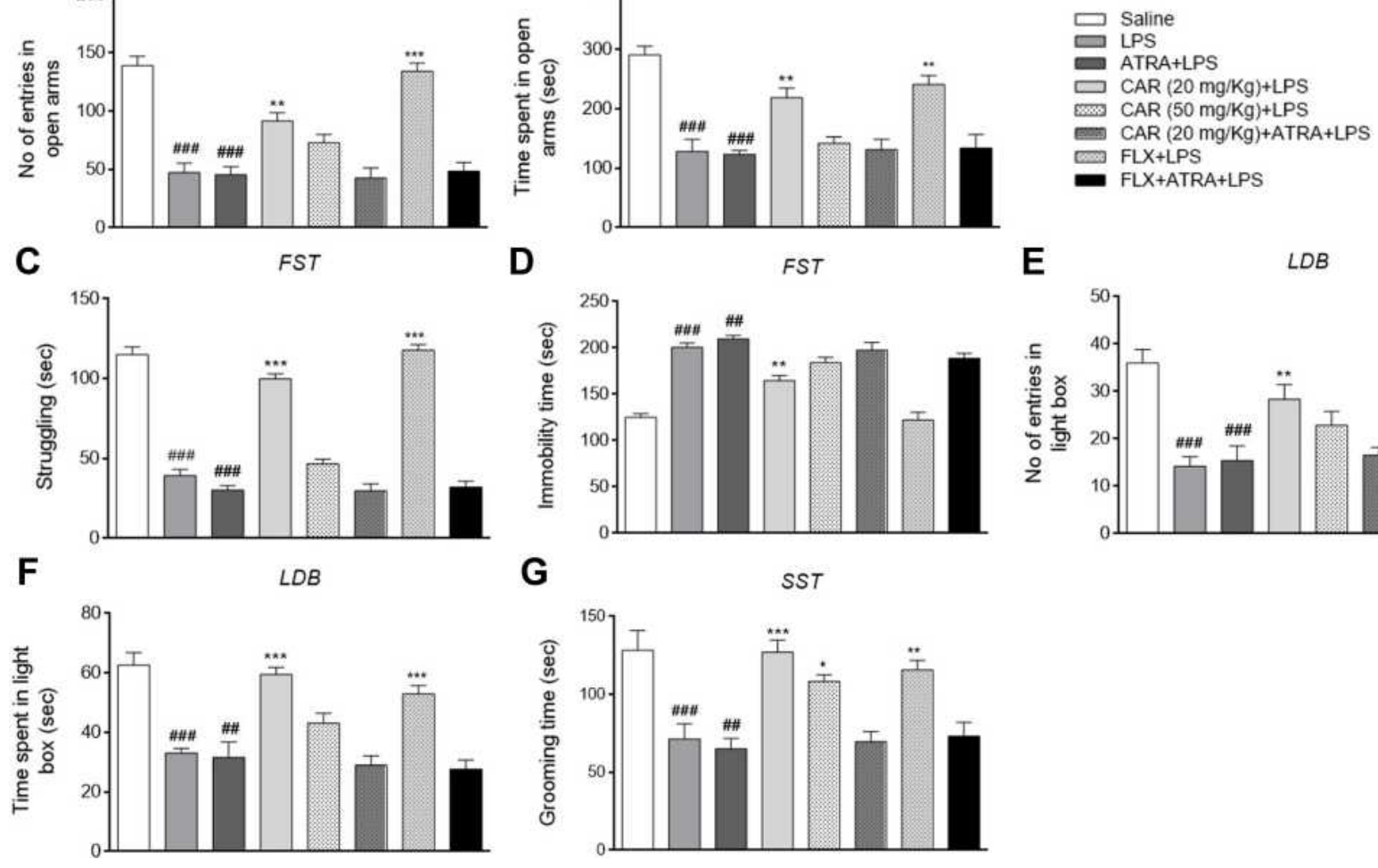

Figure 2 Effects of carvacrol (CAR) on LPS-induced behavioral deficits. Effect of CAR and LPS on the EPM test (A and B), FST (C and D), LDB test (E and F), and SST (G). Data are expressed as means \pm SEM and analyzed by one-way ANOVA followed by Bonferroni multiple comparisons test using GraphPad Prism 6 software. Saline, LPS, CAR $+L P S$, and FLX+LPS groups represent the first cohort ( $n=10 /$ group), while the LPS+ATRA, CAR+ATRA+LPS, and FLX+ATRA+LPS were from the second cohort ( $n=8 /$ group). ${ }^{\#} p<0.00 \mathrm{I}$ and $\# p<0.0 \mathrm{I}$ indicates a significant difference compared to the saline group and $* p<0.05, * * p<0.01$, $* * * p<0.001$ is compared to the LPS group. Abbreviations: CAR, carvacrol; LPS, lipopolysaccharide; ATRA, all-trans retinoic acid; FLX, fluoxetine; EPM, elevated plus maze; FST, forced swim test; LDB, light-dark box; SST, sucrose splash test. 
$* * p<0.01,20 \mathrm{mg} / \mathrm{kg})$. Furthermore, carvacrol increased the struggling capacity (Figure $2 \mathrm{C}, * * * p<0.001$ ) and alleviated immobility (Figure $2 \mathrm{D}, * * p<0.01$ ) in the FST. Similarly, the $20 \mathrm{mg} / \mathrm{kg}$ dose of carvacrol increased the number of entries (Figure $2 \mathrm{E}, * * p<0.01$ ) and time spent in the light compartment (Figure $2 \mathrm{~F}, * * * p<0.001$ ), and was associated with a higher grooming time (Figure $2 \mathrm{G}$, $* * * p<0.001)$ in the SST. Treatment with the $50 \mathrm{mg} / \mathrm{kg}$ dose of carvacrol did not significantly influence the measures obtained in the EPM test, LDB test, or FST, but led to an extended grooming time in the SST (Figure 2G, $\left.{ }^{*} p<0.05\right)$. Moreover, co-treatment with LPS and ATRA further exacerbated the anxiety-like behavioral deficits, whereas treatment with carvacrol did not elicit any ameliorative effects on LPS-induced behavioral deficits in ATRA-treated groups (second cohort).

\section{Effects of Carvacrol on LPS-Induced Histopathological Changes}

HE staining results revealed significant histopathological changes in LPS-treated animals (Figure 3, ${ }^{\# \#} p<0.001$ ).
Significant alterations were observed in the LPS group compared to saline-treated animals. The saline group showed a normal pyramidal cell shape with a well-demarcated round nucleus (Figure 3). There was no vacuolization or lipid globules, and all neurons exhibited a relative basophilic cytoplasm. Nevertheless, many histopathological alterations were observed in the LPS-treated groups, including multiple vacuoles. Many neurons lacked dendrites and were encircled by peri-cellular halos (Figure 3). Carvacrol administration caused marked mitigation of these changes. Therefore, a high degree of cellular integrity was evident in the carvacrol (20 mg/kg)-treated group (Figure 3, cortex: $* * * p<0.001$, CA3: $* * p<0.01)$. Nevertheless, carvacrol pretreatment in the ATRA-treated groups did not result in any improvement of the LPS-induced histopathological changes.

\section{Effects of Carvacrol on LPS-Induced Apoptosis}

We further demonstrated the anti-apoptotic action of carvacrol by measuring the level of p-JNK in the cortex of all treated groups (Figure 4A). LPS treatment increased p-JNK
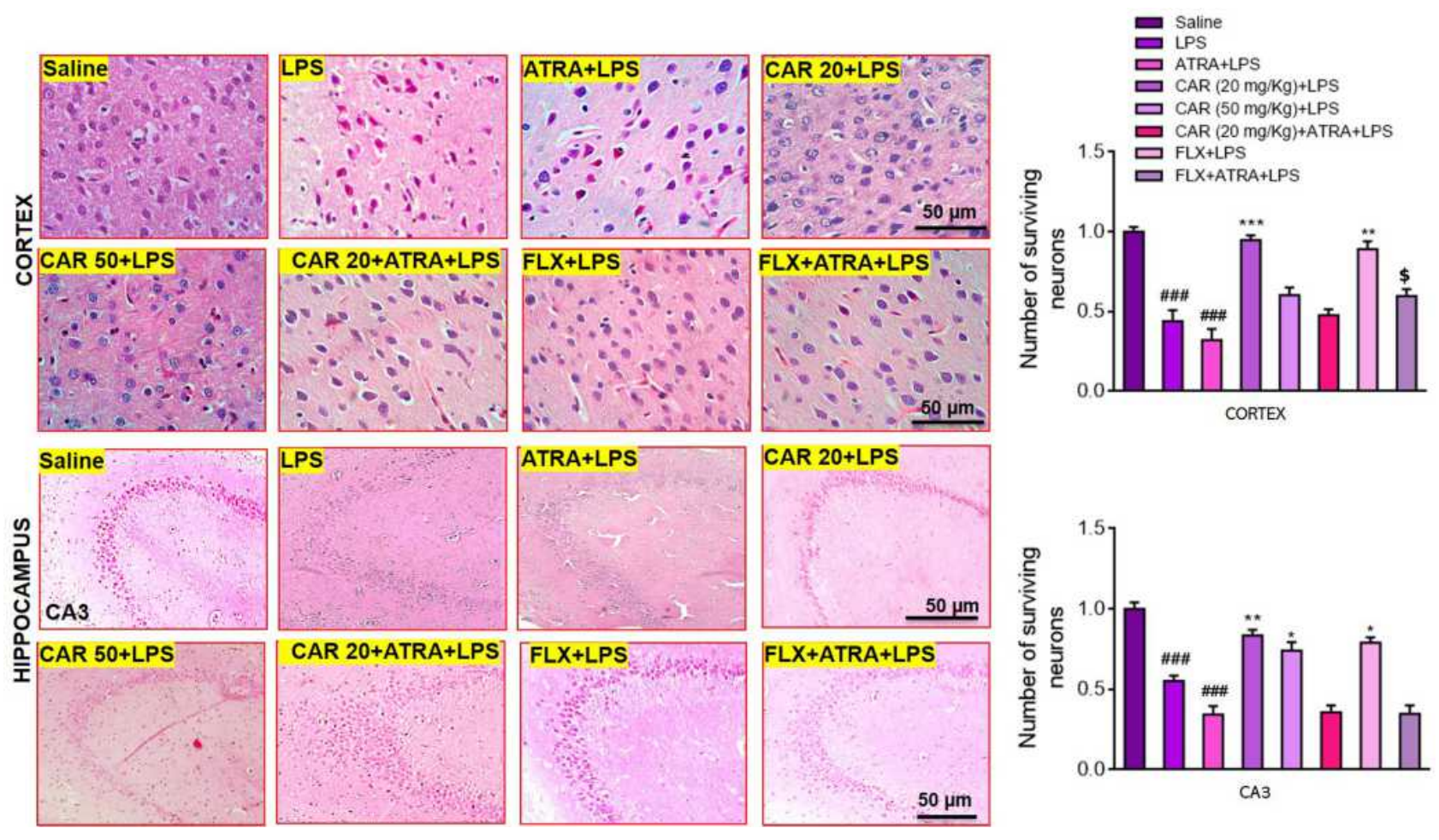

Figure 3 Hematoxylin and eosin (H\&E) staining showing the extent of surviving neurons in the cortex and hippocampus (Corno amonus, CA). Scale bar 50 um, magnification $40 \times$. Dead neurons were characterized by a swollen cytoplasm, vacuolization, scalloped morphology with intense cytoplasmic eosinophilia, and nuclear basophilia. Data are expressed as means \pm SEM. ${ }^{\#} p<0.001$ indicates a significant difference compared to the saline group, ${ }^{*} p<0.05, * * p<0.01$, $* * * p<0.001$ is compared to the LPS group and $\$ p<0.05$ is compared to ATRA+LPS group. CAR 20: carvacrol (20 mg/kg); CAR 50: carvacrol (50 mg/kg); LPS: lipopolysaccharide; ATRA: all-trans retinoic acid; FLX: fluoxetine. The $\mathrm{H} \& \mathrm{E}$ slides were made after the euthanization of animals following behavioral analysis. The saline, $L P S$, CAR+LPS, and FLX+LPS groups were those studied in the first cohort ( $n=5 /$ group), while the LPS+ATRA, CAR+ATRA+LPS, and FLX+ATRA+LPS groups were from the second cohort ( $n=4 / g r o u p$ ). 
levels (Figure 4A, ${ }^{\# \#} p<0.01$ ), while carvacrol pretreatment attenuated this increase (Figure $4 \mathrm{~A}, * * p<0.01$ ). To further validate the expression of apoptotic marker caspase- 3 and anti-apoptotic marker Bcl-2, we measured their expression in the cortex and hippocampus by immunohistochemistry analysis. The expression of the anti-apoptotic factor Bcl-2 was markedly decreased in both the cortex and hippocampus of the LPS-treated group (Figure 4B, ${ }^{\# \# \#} p<0.001$ ). Consistently, the expression of the pro-apoptotic factor caspase- 3 was upregulated in the LPS-treated group (Figure 4C, \#\#\# $p<0.001)$. In line with the results of $\mathrm{p}$-JNK, a significant upregulation of the anti-apoptotic factor $\mathrm{Bcl}-2$ was seen in carvacrol-treated rats (Figure $4 \mathrm{~B}$, cortex: $* * p<0.01, \mathrm{CA} 3$ : $* * p<0.01$, DG: $* * * p<0.001)$, with a significant decrease in the expression of proapoptotic caspase-3 factor in carvacroltreated rats (Figure 4C, cortex: $* * p<0.01$, CA3: $* * * p<0.001$, DG: $* * * p<0.001$ vs LPS group). However, carvacrol administration did not attenuate LPS-induced apoptosis in the ATRA-treated groups.

\section{Effects of Carvacrol on LPS-Induced Neuroinflammation}

Due to the significant involvement of inflammatory mediators in depression, we sought to determine whether carvacrol treatment can affect neuroinflammation. Therefore, we studied the expression of TNF- $\alpha, \mathrm{COX}-2$, and $\mathrm{p}-\mathrm{NFkB}$ in the cortex by ELISA. The levels of both TNF- $\alpha$ and COX-2 in the LPS group were significantly increased compared to the saline group (Figure $5 \mathrm{~A}$ and $\mathrm{B},{ }^{\# \#} p<0.001$ ). Consistent with the upregulated levels of $\mathrm{TNF}-\alpha$ and $\mathrm{COX}-2$, the expression of p-NFkB was also markedly increased in the

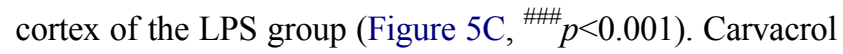
pretreatment induced a significant downregulation of TNF$\alpha$ (Figure 5A, $* \mathrm{p}<0.05$ ), COX-2 (Figure 5B, $* * * p<0.001$ ), and $\mathrm{p}-\mathrm{NFkB}$ (Figure $5 \mathrm{C}, * * \mathrm{p}<0.01$ ) expression in the cortex of treated animals relative to the LPS group. For further validation, we performed immunohistochemical analysis, and the results revealed overexpression of TNF- $\alpha$ and $\mathrm{p}-\mathrm{NFkB}$ in both the cortex and hippocampus of LPS-treated

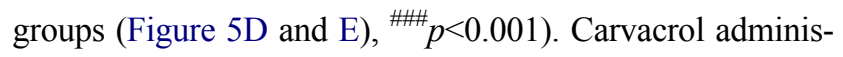
tration reduced the LPS-induced overexpression of TNF- $\alpha$ in the cortex (Figure 5D, $* * * p<0.001$ ) and hippocampus (Figure 5D, $* * p<0.01$ ). Similarly, carvacrol also attenuated the LPS-induced overexpression of $\mathrm{p}-\mathrm{NFkB}$ in the cortex (Figure 5E, $* * * p<0.001$ ) and hippocampus (Figure 5E, $* * p<0.01)$. Moreover, co-treatment with LPS and ATRA further exaggerated the neuroinflammatory markers, while treatment with carvacrol did not reverse the detrimental effects of LPS in ATRA-treated groups.

\section{Effects of LPS and Carvacrol on the Expression of Nrf2 and Nrf2 Dependent Downstream Antioxidant Protein HO-I}

The antioxidant mechanism of carvacrol was explored by measuring the expression of $\mathrm{Nrf} 2$ and the $\mathrm{Nrf} 2$ downstream signaling protein HO-1. Western blot analysis demonstrated that the Nrf2 expression level in the LPStreated group was significantly reduced compared to that in the saline group (Figure $6 \mathrm{~A},{ }^{\#} p<0.05$ ). Consistent with the downregulated expression of $\mathrm{Nrf} 2$, expression of the downstream antioxidant protein HO-1 was also markedly decreased in the cortex of the LPS-treated group in comparison to the saline group (Figure 6A, ${ }^{\#} p<0.05$ ). Carvacrol administration induced significant upregulation of Nrf2 and HO-1 in the cortex of treated animals relative to the LPS group (Figure $6 \mathrm{~A}, * p<0.05$ ). To further validate, a noticeable upregulation was also observed for $\mathrm{Nrf} 2$ and HO-1 using ELISA analysis (Figure 6B, $* * * p<0.001$ and $* * p<0.01)$. Nonetheless, ATRA-treated groups did not exhibit a carvacrol-mediated upregulation of Nrf2 or HO1. Thus, these results provide a clear indication that carvacrol might possess an antioxidant action via activation of the Nrf2/HO-1 signaling pathway.

\section{Effects of Carvacrol Pretreatment on LPS-Induced Lipid Peroxidation and Antioxidant Enzymes}

To determine the neuroprotective role of carvacrol against LPS-evoked lipid peroxidation and oxidative stress, we measured the levels of catalase, GSH, GST, and thiobarbituric acid reactive substances (TBARS) in both the hippocampus and prefrontal cortex. Carvacrol administration ameliorated oxidative stress and restored the expression of antioxidant enzymes in both the hippocampus and prefrontal cortex to varying degrees. Catalase, GSH, and GST levels in the LPS-treated group were significantly lower than those in the saline group (Figure 7A-F), ${ }^{\# \# \#} p<0.001$ ). On the other hand, the level of TBARS showed a marked elevation in both the hippocampus and prefrontal cortex of LPS-treated rats compared to the saline group (Figure $7 \mathrm{G}$ and $\left.\mathrm{H},{ }^{\# \#} p<0.001\right)$. Carvacrol treatment induced the production of catalase, GSH, and GST in both the cortex and hippocampus (Figure 7A and B, $* * p<0.01$; Figure $7 \mathrm{C}$ and $\mathrm{D},{ }^{*} p<0.05$ and $* * * p<0.001$; and Figure $7 \mathrm{E}$ and $\mathrm{F}$, 
A

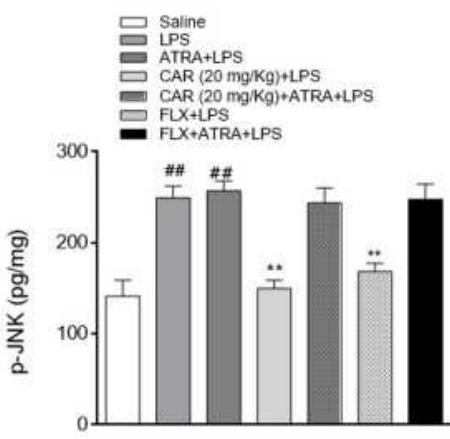

B
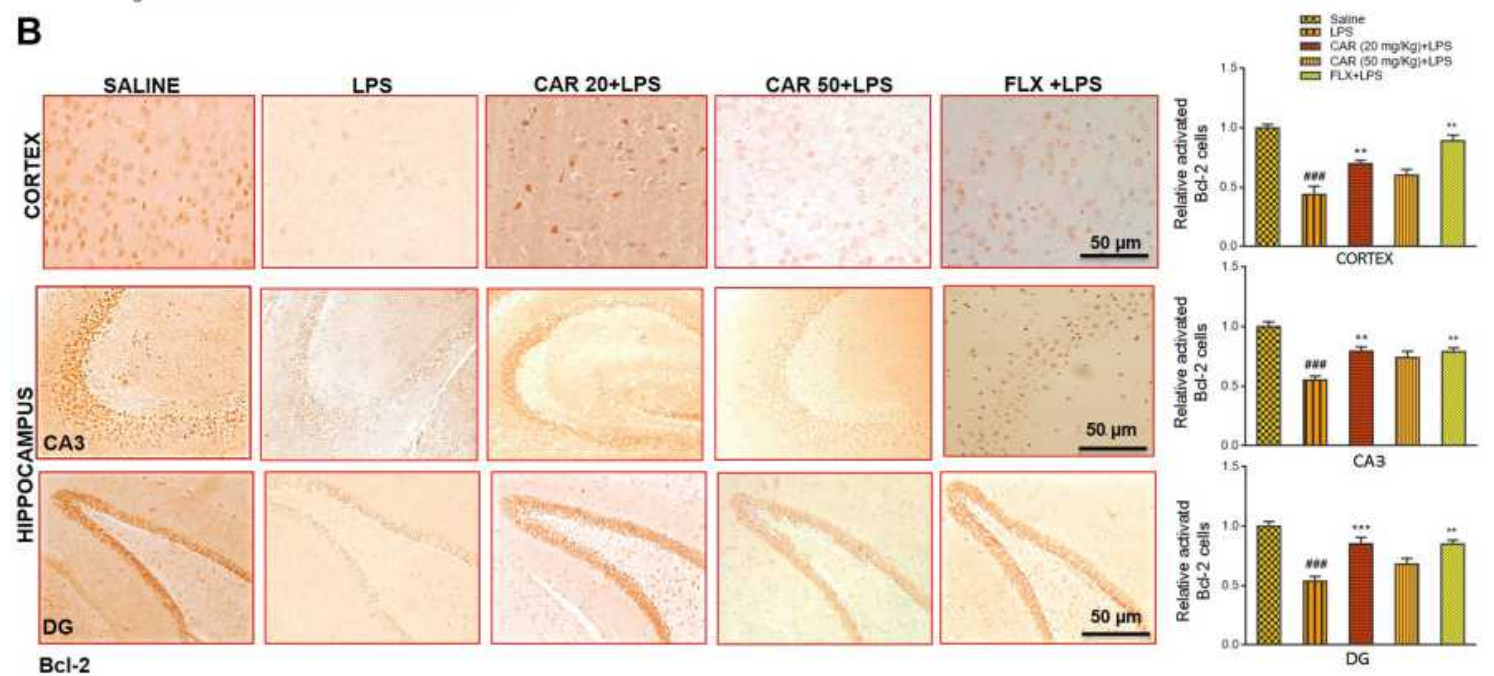

C
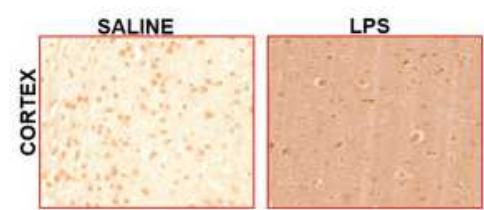

CAR 20+LPS
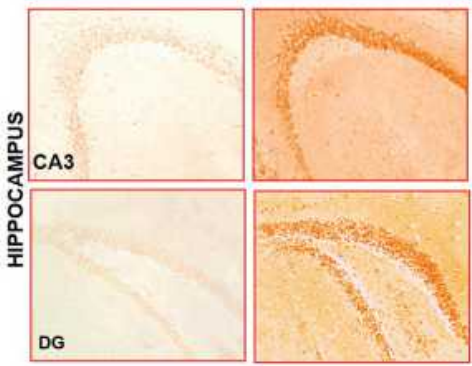

Caspase-3
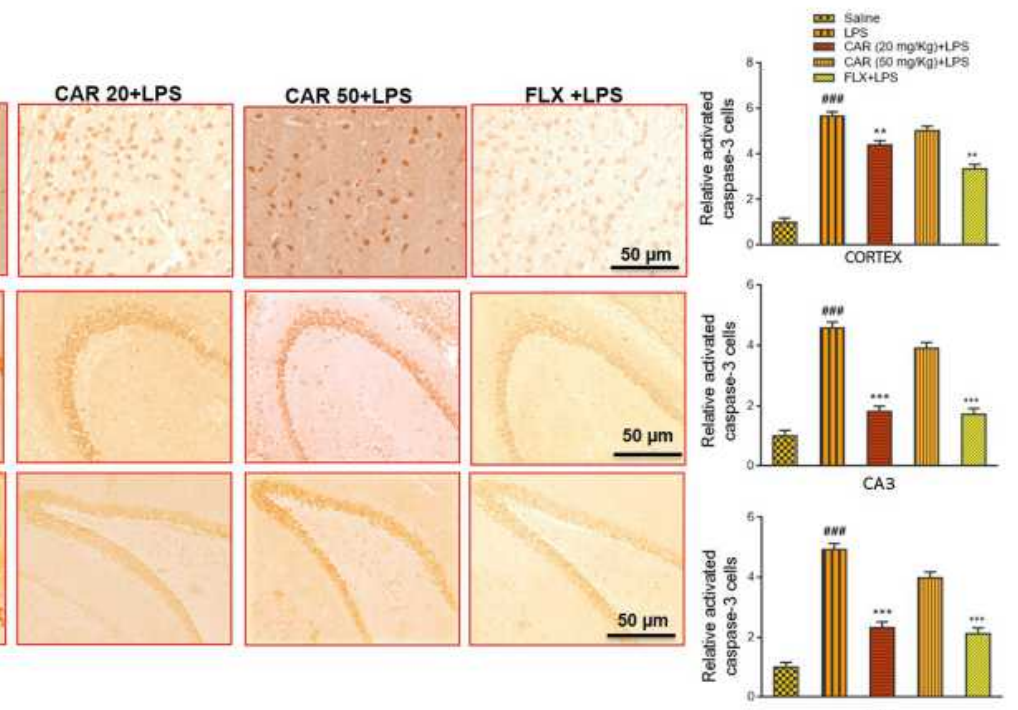

DG

Figure 4 Effects of carvacrol on LPS-induced neuronal apoptosis. (A) $\mathrm{P}$-JNK levels were measured by ELISA. Data are expressed as means \pm SEM. \#\# $p<0.00 \mathrm{I}$ indicates a significant difference compared to the saline group and ${ }^{* *} p<0.01$ is compared to the LPS group. (B) Immunohistochemistry results for Bcl-2 in the cortex and hippocampus. Scale bar $50 \mu \mathrm{m}$, magnification $40 \times$. Data are expressed as means \pm SEM. ${ }^{\# \#} p<0.001$ indicates a significant difference compared to the saline group and $*_{*}^{*} p<0.01$, *** $p<0.00$ I is compared to the LPS group. (C) Immunohistochemistry results for caspase-3 in the cortex and hippocampus. Scale bar $50 \mu \mathrm{m}$, magnification $40 \times$. Data are expressed as means \pm SEM. ${ }^{\# \#} p<0.001$ and \#\# $p<0.01$ indicates a significant difference compared to the saline group and $* * p<0.01$, $* * * p<0.001$ is compared to the LPS group. The immunohistochemistry sections were prepared after the euthanization of animals following behavioral analysis. The Saline, LPS, CAR+LPS, and FLX+LPS groups were taken as the first cohort ( $n=5 /$ group), while the LPS+ATRA, CAR+ATRA+LPS, and FLX+ATRA+LPS groups were from the second cohort ( $n=4 / g r o u p)$. For ELISA analysis, animals were taken from both the first cohort ( $n=5 /$ group) and the second cohort ( $n=4 /$ group).

Abbreviations: CAR 20, carvacrol (20 mg/kg); CAR 50, carvacrol (50 mg/kg); LPS, lipopolysaccharide; ATRA, all-trans retinoic acid; FLX, fluoxetine; P-JNK, Jun N-terminal kinase; Bcl-2, B cell lymphoma-2. 


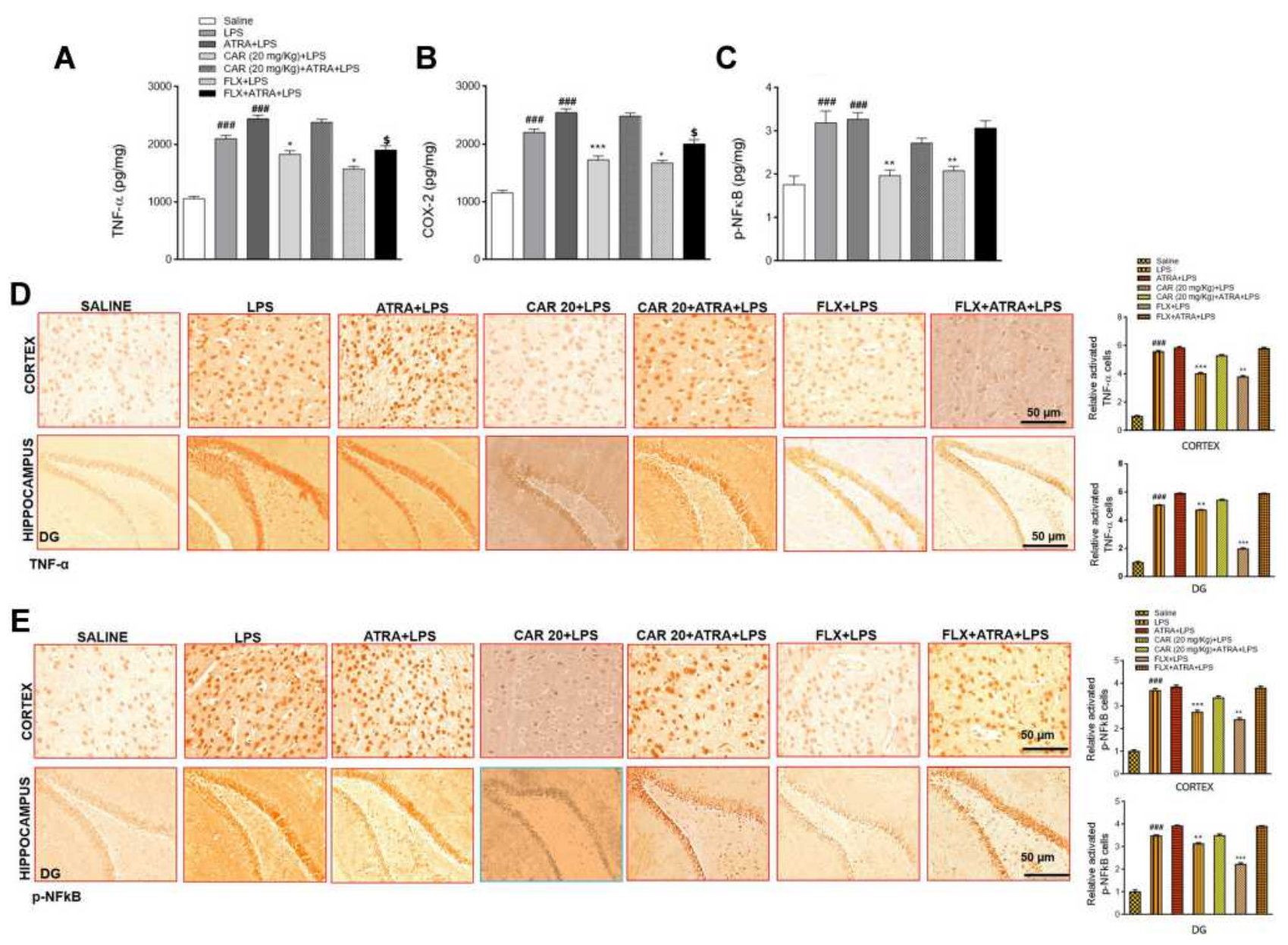

Figure 5 Effects of carvacrol on LPS induced neuroinflammation. (A) TNF- $\alpha$ levels, (B) COX-2 levels, and (C) p-NFkB levels were measured by ELISA. Data are expressed as means \pm SEM. ${ }_{p}<0.001$ indicates a significant difference compared to the saline group, ${ }_{p}^{*}<0.05, * * p<0.01$ and $* * * p<0.001$ is compared to the LPS group and ${ }^{\$} p<0.05$ is compared to ATRA+LPS group. (D) Immunohistochemistry results for TNF- $\alpha$ in the cortex and hippocampus. (E) Immunohistochemistry results for p-NFkB in the cortex and hippocampus. Scale bar $50 \mu \mathrm{m}$, magnification $40 x$. Data are expressed as means \pm SEM. ${ }^{m} p<0.001$ indicates a significant difference compared to the saline group and $* * p<0.01$, *** $p<0.001$ is compared to the LPS group.

Abbreviations: CAR 20, carvacrol (20 mg/kg); CAR 50, carvacrol (50 mg/kg); LPS, lipopolysaccharide; ATRA, all-trans retinoic acid; FLX, fluoxetine; TNF- $\alpha$, tumor necrosis factor-alpha; COX-2, cycloxygenase-2); p-NFkB, nuclear factor-kappa B.

${ }^{*} p<0.05$, and $\left.*^{*} p<0.01\right)$. However, a noticeable decline was observed in the TBARS level of carvacrol-treated rats in both the cortex and hippocampus compared to the LPS group (Figure $7 \mathrm{G}$ and $\mathrm{H},{ }^{* *} p<0.01$ ). Further, carvacrol pretreatment in the ATRA-treated groups did not reveal any antioxidant or free-radical scavenging effects (Figure 7), validating the Nrf2 results (Figure 6). Our results demonstrated that carvacrol might participate in free radical scavenging to ameliorate LPS-induced oxidative stress.

\section{Molecular Docking}

The above-mentioned results suggest that carvacrol activates Nrf2 signaling, which is usually kept inactive when bound to the downstream signaling protein Keap1.
Therefore, we conducted a docking analysis of carvacrol and Keap1 to explore the possible affinity of carvacrol for binding Keap1 to interfere with the interaction between Keap1 and Nrf2, thus facilitating the translocation of Nrf2 into the nucleus. Computational studies revealed efficient binding of carvacrol with Keap1 and its orientation into the Nrf2 binding pocket. The Nrf2 binding pocket designated for Keap1 is shallow and it consists of three subregions: acidic, planner acceptor, and sulfamide (Figure 8). ${ }^{48}$ Hence, we first selected the appropriate subsite and binding orientation of carvacrol in Keap1. The results illustrated the orientation of carvacrol in two distinct stoichiometric conformations in the Nrf2 binding pocket of Keap1 (Figure 8B and C). Carvacrol exhibited a docking score of 35.08 and 37.14 after occupying the acidic and 


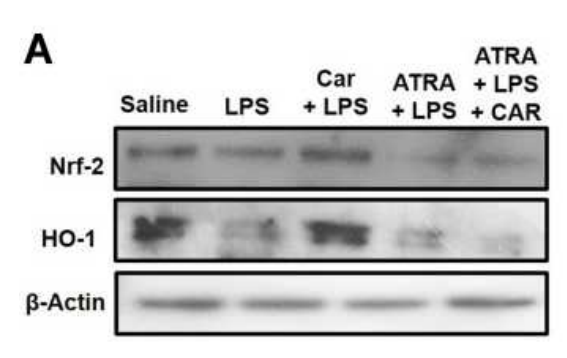

B

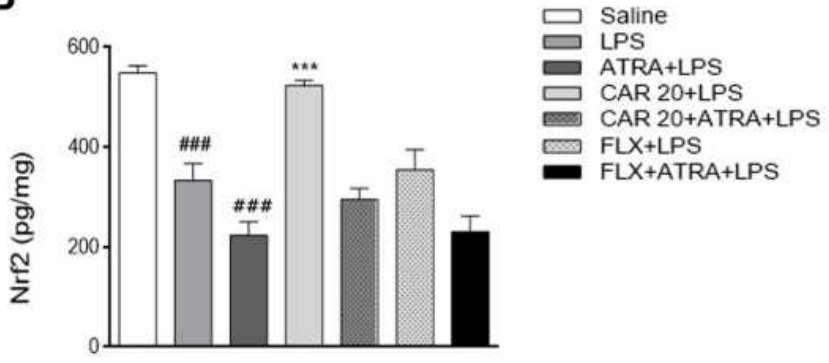

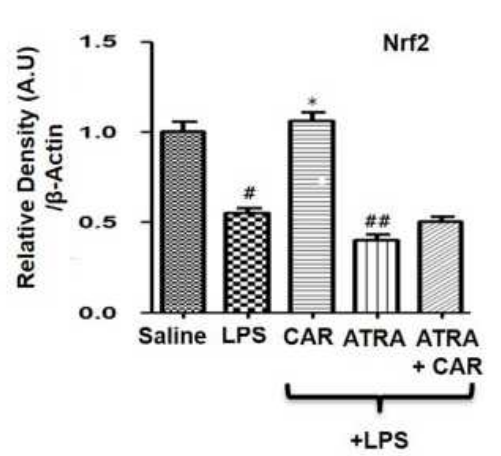
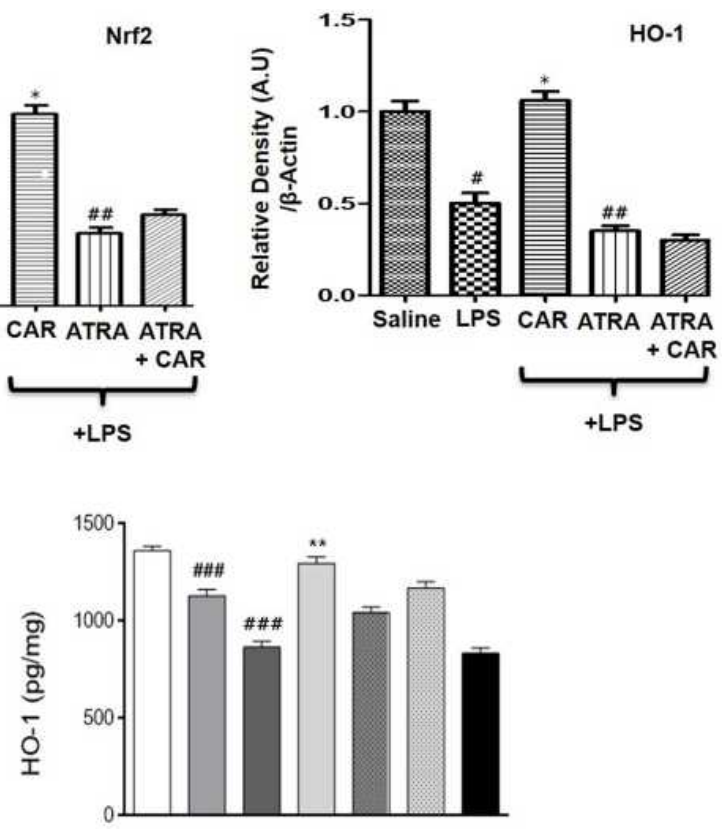

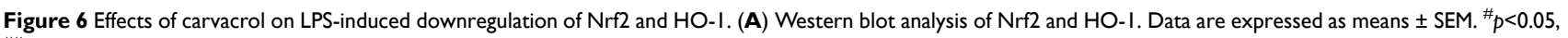
$\#^{\prime}<0.01$ compared to the saline group while $* p<0.05$ compared to the LPS group. (B) Nrf2 and HO-I levels were measured by ELISA. Data are expressed as means \pm SEM.

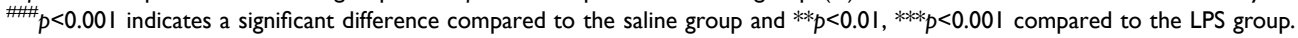

Abbreviations: CAR 20, carvacrol (20 mg/kg); CAR 50, carvacrol (50 mg/kg); LPS, lipopolysaccharide; ATRA, all-trans retinoic acid; FLX, fluoxetine; Nrf2, nuclear factor E2-related factor; HO-I, heme oxygenase I.

sulfamide regions, respectively (Figure $8 \mathrm{~B}$ and $\mathrm{C}$ ), of the Nrf2 binding pocket of Keap1 (Table 1). Moreover, the interpretation of molecular interactions observed suggested that carvacrol shared two conventional hydrogen bonds with Arg483 and Ser508 in the acidic sub-region of Keap1 (Figure 8B and E). Furthermore, carvacrol also formed a hydrogen bond with the residue SER602 in the sulfamide sub-region of Keap1 (Figure 8C and F). Overall, we propose that carvacrol orients in both the acidic and sulfamide binding pockets of Keap1 and have established hydrogen bonds with their respective binding residues Arg483, Ser508, and Ser602. In addition to forming hydrogen bonds with the active residues of Keap1, carvacrol also exhibited nonpolar interactions with binding residues of the Nrf2 binding site of Keap1 (Figure 8, Table 1). For instance, carvacrol established a nonpolar bond with Tyr525, a binding residue that has already been reported for interaction with some small molecule inhibitors of Nrf2. ${ }^{49}$ Carvacrol demonstrated $\pi-\sigma$ and alkyl interactions in the sulfamide region with residues Arg415 and Ala556, respectively (Figure $8 \mathrm{C}$ and $\mathrm{F}$ ). In addition to polar interactions with the binding residue Arg415, carvacrol also formed various nonpolar bonds including Van der Waal and hydrophobic interactions with the Nrf2 amino acid residues of the Keap1 binding pocket (Figure 8, Table 1).
Hence, we speculate that carvacrol occupies the Nrf2 binding pocket of Keap1 and reduces the interaction of Keap1 with Nrf2.

\section{Discussion}

Phytomedicine has been a great source of neuroprotective agents in several neurodegenerative diseases. Lately, the use of phytochemicals has increased; nevertheless, extensive research still needs to be conducted before phytochemicals can be approved as neuroprotectants and used for treatment in human diseases. ${ }^{50}$ Previous literature has revealed that carvacrol, a monoterpene, exhibits antioxidant, anti-inflammatory, and neuroprotective activities in animal models of neurodegeneration. ${ }^{51}$ The present study was designed to explore the antioxidant, anti-neuroinflammatory, and neuroprotective action of carvacrol against LPS-provoked anxiety and depression.

LPS-mediated depressive-like behaviors are characterized by immobility with a simultaneous decrease in struggling time. Nevertheless, carvacrol pretreatment markedly attenuated this behavioral despair by decreasing immobility and increasing struggling time. ${ }^{52}$ Previous studies demonstrated that increased immobility in the FST is mediated by proinflammatory cytokines and a build-up of reactive oxygen species (ROS) in the LPS model. ${ }^{53}$ Our 

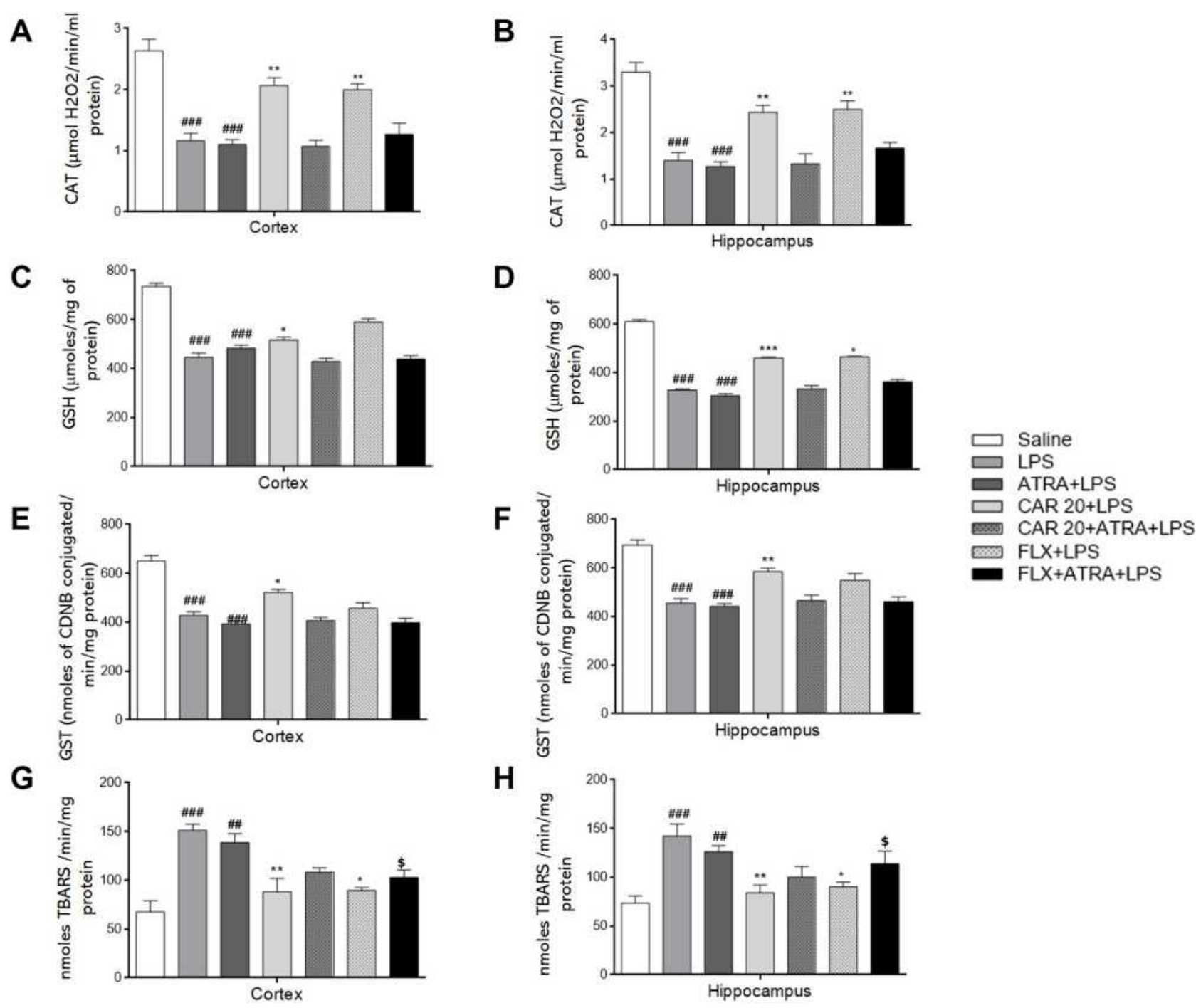

Figure 7 Effects of carvacrol pretreatment on antioxidant enzymes and LPS-induced lipid peroxidation. Effects of LPS and CAR on levels of CAT (A and B); GSH (C and D); GST (E and F), and TBARS ( $\mathbf{G}$ and $\mathbf{H}$ ). Data are expressed as means \pm SEM. $*_{p}<0.05, * * p<0.01, * * * p<0.001$ is compared to LPS group and ${ }^{\$} p<0.05$ is compared to ATRA+LPS group.

Abbreviations: CAR, carvacrol; LPS, lipopolysaccharide; ATRA, all-trans retinoic acid; FLX, fluoxetine; CAT, catalase; GSH, reduced glutathione; GST, glutathioneS-transferase; TBARS, thiobarbituric acid reactive substances.

results attested to the previously reported data, that carvacrol can mitigate depression-like symptoms. ${ }^{33}$ Unlikely, these authors also demonstrated a significant decrease in immobility time in FST at a dose (carvacrol $50 \mathrm{mg} / \mathrm{kg}$ ). The possible discrepancy may due to species difference and rate and route of carvacrol administration. Additionally, we measured the grooming behavior in the SST, which is considered an indicator of apathy and decreased motivation. LPS administration caused a noticeable reduction in grooming time, while carvacrol pretreatment increased grooming time, showing a reversal of LPS-induced apathy and lack of motivation. Our data demonstrated that carvacrol mitigates LPS-induced behavioral despair and apathy, thus improving the core symptoms of depressive-like behaviors. Furthermore, we evaluated anxiety-like behaviors by employing two frequently used paradigms, an EPM test, and an LDB test. The data obtained from the EPM and LDB tests showed the anxiolytic potential of carvacrol, as elevated values of the open arm and lightbox parameters indicate anxiolytic activity. ${ }^{54}$

Several studies have reported the involvement of ROS in the pathophysiology of neurodegenerative diseases. ${ }^{55,56}$ Likewise, oxidative stress can exacerbate depression as the 
A
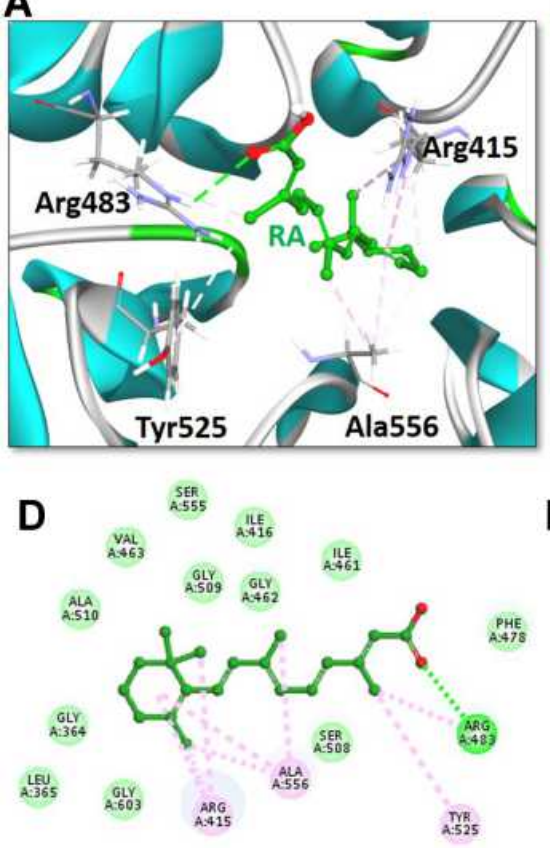

B

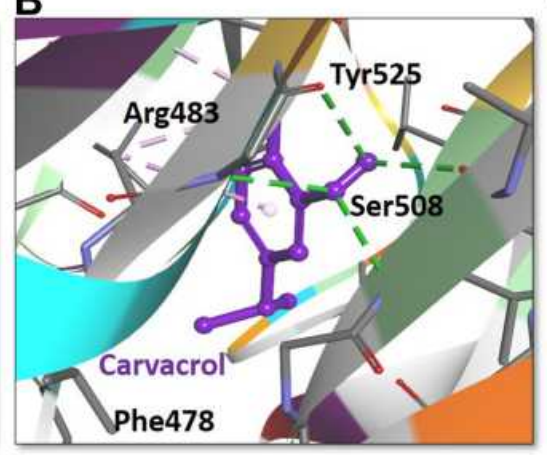

E

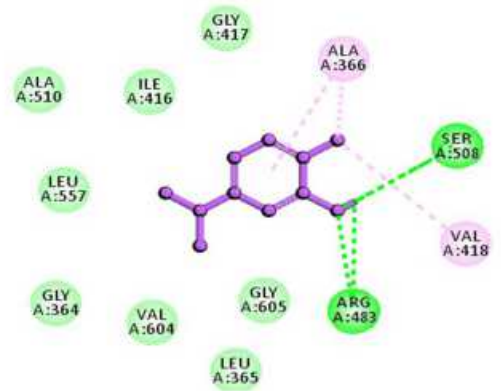

C

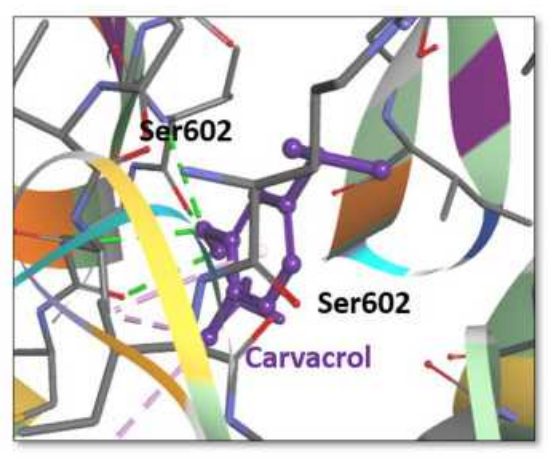

$\mathbf{F}$

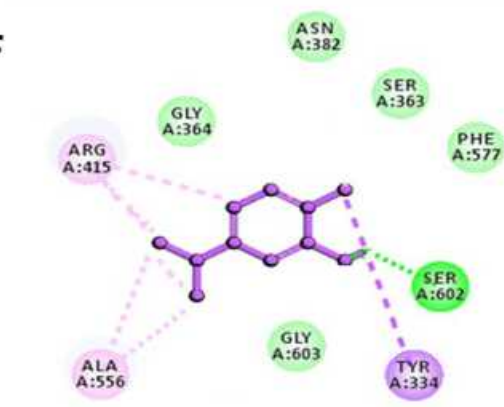

Figure 8 Retinoic acid and carvacrol bound to the Nrf2-binding site of Keap-I. The upper panel $(\mathbf{A}-\mathbf{C})$ illustrates the binding orientation of retinoic acid and carvacrol in the Nrf2-binding site of Keap-I. A) Retinoic acid formed a conventional hydrogen bond with binding residue Arg483 of Keap-I. B) One carvacrol molecule established a hydrogen bond with two binding residues Arg83 and Ser508 in the acidic region of Keap-I. C) Another carvacrol molecule constituted a hydrogen bond with amino acid Ser602 in the sulfamide region of Keap-I. Retinoic acid and carvacrol are displayed as ball-and-stick models and are assigned green and purple colors, respectively. Keap-I binding residues participating in either polar or non-polar interactions are represented as stick models. Conventional hydrogen bonds are illustrated as dotted lines in green color. Other non-polar bonds are depicted as light pink-colored dotted lines. The lower panel (D-F) shows the two-dimensional interaction pattern of retinoic acid and carvacrol with Keap-I. D) Retinoic acid and E) carvacrol in the acidic region of Keap-I, and F) carvacrol in the sulfamide region of Keap-I show the established hydrogen bonds with Keap-I. Retinoic acid and carvacrol are portrayed as ball-and-stick models and colored as green and purple, respectively. Hydrogen bonds forming binding residues are illustrated in the form of labeled circles in green color. Conventional hydrogen bonds are demonstrated as green-colored dotted lines. Other hydrophobic binding residues are shown as labeled circles in light green color (Van der Waals interactions) and light pink color (non-polar interactions).

brain has limited anti-oxidants combating capacity. ${ }^{57,58}$ This notion is further supported by the fact that several clinically antidepressant drugs alleviated ROS in depressed patients. ${ }^{59}$ The transcription factor Nrf2 and its downstream signaling protein HO-1 constitute the major cellular antioxidant system that regulates the gene expression of several cytoprotective antioxidants. Our results demonstrated that LPS stimulated the production of ROS and led to an imbalance between oxidants and endogenous antioxidants, and thus resulted in the build-up of oxidative stress similar to that seen in previous studies. ${ }^{60}$ Modulation of the Nrf2 pathway is considered

Table I Docking Scores and Hydrophobic Interactions of Retinoic Acid and Carvacrol with Keap-I

\begin{tabular}{|l|l|l|l|l|l|l|}
\hline \multirow{2}{*}{ Inhibitor } & \multirow{2}{*}{$\begin{array}{l}\text { Docking } \\
\text { Score }\end{array}$} & \multicolumn{3}{|l|}{ Hydrogen Bonds (<3.5A) } & \multirow{2}{*}{ Hydrophobic Interactions } \\
\cline { 3 - 6 } & $\begin{array}{l}\text { Amino } \\
\text { Acid }\end{array}$ & $\begin{array}{l}\text { Amino } \\
\text { Acid } \\
\text { Atom }\end{array}$ & $\begin{array}{l}\text { Ligand } \\
\text { Atom }\end{array}$ & $\begin{array}{l}\text { Distance } \\
(\mathbf{A})\end{array}$ & \\
\hline Retinoic Acid & 52.69 & Arg483 & HE & O2 & 2.8 & $\begin{array}{l}\text { Arg415, Ala556, Tyr525, Ser508, Phe478, Ile46I, Gly462, Ile4I6, } \\
\text { Gly509, Ser555, Val463, Ala510, Gly364, Leu365, Gly603 }\end{array}$ \\
\hline Carvacrol A* & 35.08 & $\begin{array}{l}\text { Arg483 } \\
\text { Ser508 }\end{array}$ & $\begin{array}{l}\text { HE } \\
\text { OG }\end{array}$ & $\begin{array}{l}\text { OI } \\
\text { H25 }\end{array}$ & $\begin{array}{l}2.3 \\
1.8\end{array}$ & Tyr525, Phe478, Arg4I5, Gly509, Ser555, GIn530 \\
\hline Carvacrol S & 37.14 & Ser602 & OG & H25 & 1.7 & Arg415, Ala556, Tyr334, Gly603, Phe577, Ser363, Asn382, Gly364 \\
\hline
\end{tabular}

Notes: *Carvacrol in acidic region; ${ }^{\#}$ Carvacrol in sulfamide region

Abbreviations: Arg, arginine; Ala, alanine; Gln, glutamine; Gly, glycine; lle, iso-leucine; Leu, leucine; Phe, phenylalanine; Ser, serine; Tyr, tyrosine; Val, valine. 
to play a pivotal role in designing strategies for cellular protection. ${ }^{19}$ Furthermore, our results are in line with previous studies indicating the downregulation of both Nrf2 and its downstream protein HO-1 in a depression model. ${ }^{61}$ Interestingly, increased levels of ROS and LPO, along with the downregulation of $\mathrm{Nrf} 2 / \mathrm{HO}-1$, were markedly recovered in carvacrol-treated animals, validating our proposed hypothesis that carvacrol exerts free-radical scavenging and antioxidant activity. We previously reported the antioxidant effect of a similar monoterpene carveol in ischemic stroke by targeting the Nrf2 ${ }^{25}$ To rule out the possible pathway of carvacrol-mediated neuroprotection, we treated LPS-intoxicated rats with both carvacrol and ATRA. As per our expectation, ATRA abrogated the neuroprotective effects of carvacrol by blocking the Nrf2 and HO-1 signaling pathways, and further, we noticed an increase in LPO and ROS levels along with a decrease in antioxidant enzymes (GST, GSH, and catalase) compared to the LPS-only group. These effects suggest that carvacrol could reduce depressive- and anxiety-like symptoms by mediating the Nrf2/HO-1 pathway. In our previous study, we demonstrated that ATRA increased infarction area by mediating neuroinflammation in the ischemic stroke model possibly by antagonizing the Nrf2 pathway. $^{21}$ Furthermore, Nrf2 can also modulate the level of serotonin, noradrenaline, and dopamine, which are the key targets of the monoamine hypothesis. A significant reduction in the level of these neurotransmitters was shown along with an elevated level of glutamate in Nrf2 $\mathrm{KO}$ mice. ${ }^{28}$

Activation of $\mathrm{Nrf} 2$ signaling inhibits the release of proinflammatory cytokines and chemokines and downregulates the p-NFkB pathway. Consistent studies implicated inflammatory processes in the pathophysiology of depression not only in laboratory animals but also in meta-analysis and postmortem brain samples. ${ }^{62,63}$ Moreover, Yao et al, found a higher level of inflammatory markers in Nrf2 null mice, and which further demonstrated higher immobility time (FST). ${ }^{64}$ In another study, Nrf2 KO mice demonstrated decrease grooming time in $\mathrm{SST}^{28}$ which supports our hypothesis here that Nrf2 antagonism could exacerbate depression (Figure 2). p-NFkB is a pleiotropic transcriptional mediator that controls multiple genes associated with inflammatory cascades and thus induces several inflammatory cytokines. ${ }^{65}$ Our findings showed that carvacrol considerably reduced the LPS-induced expression of $\mathrm{p}-\mathrm{NFkB}, \mathrm{TNF} \alpha$, and COX2. HO-1, a downstream target protein of $\mathrm{Nrf2}$, can inhibit proinflammatory cytokines. ${ }^{66,67}$ Moreover, activated Nrf2 migrates into the nucleus, leading to reduced expression of inflammatory mediators, including $\mathrm{COX} 2{ }^{66}$ Carvacrol significantly decreased the inflammatory mediators accompanied by an upregulated expression of the antioxidant HO-1. Both of these effects were abolished in ATRA-treated groups, thus supporting our hypothesis that carvacrol can play an

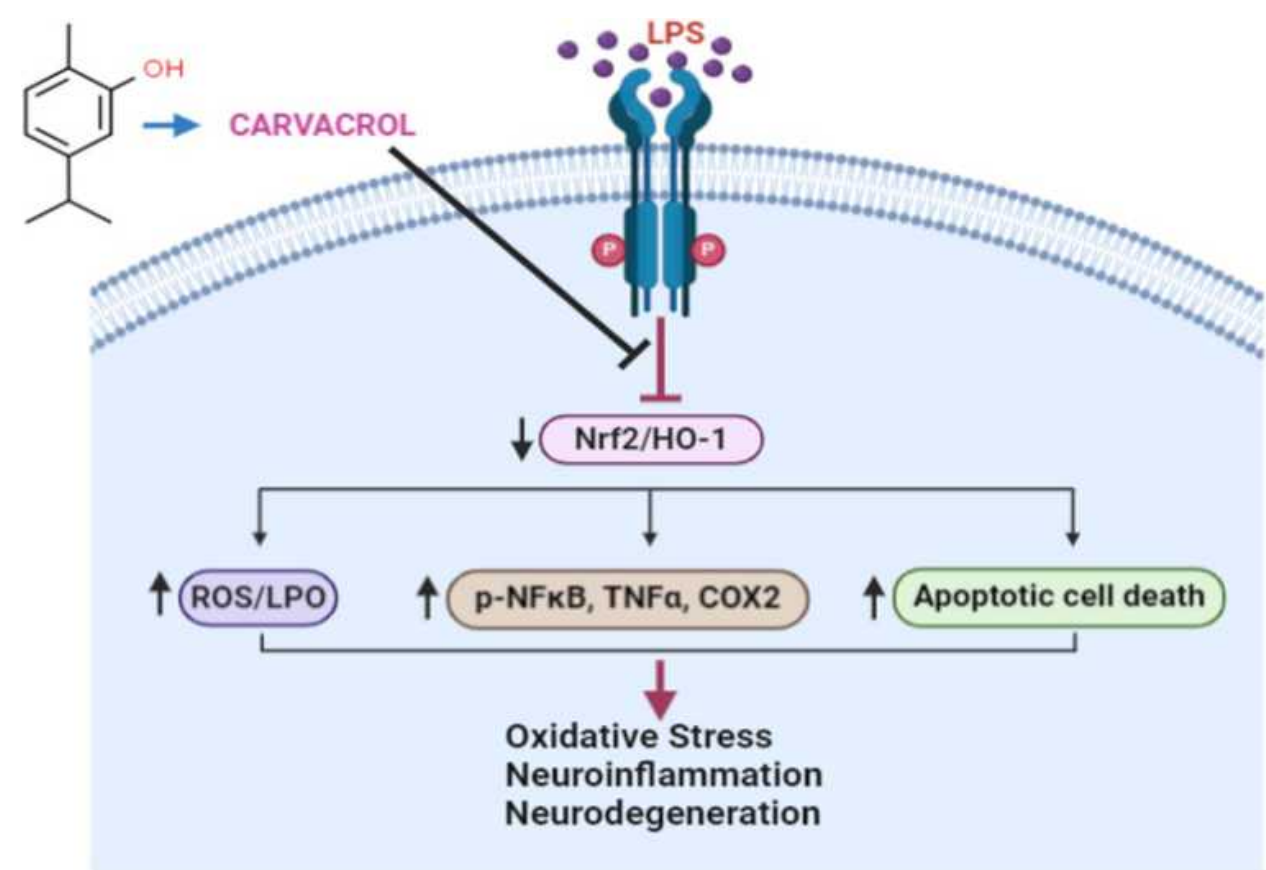

Figure 9 A proposed pathway for the effects of carvacrol pretreatment on LPS-induced oxidative stress, neuroinflammation, and neurodegeneration. 
anti-neuroinflammatory role via activation of the $\mathrm{Nrf} 2 /$ HO-1 pathway. Moreover, treatment with ATRA further elevated the level of inflammatory markers, and as such can exacerbate the depression symptoms due to compromised synthesis of dopamine and serotonin. ${ }^{28}$

Consistent studies have reported the involvement of MAP kinases, notably JNK, in the propagation of several degenerative disorders. Moreover, the JNK pathway can be activated by multiple factors, including ROS and proinflammatory mediators. ${ }^{68}$ Likewise, a close interplay of caspase-3 and JNK is implicated in the execution of the mitochondrial apoptotic pathway in several neurodegenerative disorders. ${ }^{69,70}$ Our data demonstrated a considerably decreased expression of anti-apoptotic $\mathrm{Bcl} 2$ as well as a significant increase in the expression of JNK and caspase-3 in LPS-injected rats. Carvacrol exhibited a substantial reduction in LPS-provoked elevation of pro-apoptotic proteins and p-JNK associated with increased anti-apoptotic mediators.

\section{Conclusion}

In summary, our in vivo results demonstrated that carvacrol could be a potent antioxidant and anti-neuroinflammatory agent that mediates neuroprotective properties in LPS-induced depressive- and anxiety-like behaviors. Further, our proposed neuroprotective mechanism suggests that carvacrol may activate the master endogenous antioxidant protein Nrf2 and may be associated with negative modulation of p-JNK and other neuroinflammatory mediators (Figure 9), and thus may offer a new therapeutic option for preventing and managing oxidative stress and neuroinflammation in neurodegenerative disorders such as depression and anxiety.

\section{Author Contributions}

All authors made substantial contributions to conception and design, acquisition of data, or analysis and interpretation of data; took part in drafting the article or revising it critically for important intellectual content; agreed to submit to the current journal; gave final approval of the version to be published, and agree to be accountable for all aspects of the work.

\section{Funding}

This project was partially supported by the grant provided by the Office of Research, Innovation, and Commercialization (ORIC) Riphah Institute of
Pharmaceutical Sciences (RIPS) Riphah International University Islamabad Pakistan.

\section{Disclosure}

The authors report no conflicts of interest for this work.

\section{References}

1. Paykel ES. Depression: major problem for public health. Epidemiol Psichiatr Soc. 2006;15(1):4-10. doi:10.1017/S1121189X00001974

2. GBD 2017 Disease and Injury Incidence and Prevalence Collaborators. Global, regional, and national incidence, prevalence, and years lived with disability for 354 diseases and injuries for 195 countries and territories, 1990-2017: a systematic analysis for the Global Burden of Disease Study 2017. Lancet. 2018.

3. Menken M, Munsat TL, Toole JF. The global burden of disease study: implications for neurology. Arch Neurol. 2000;57(3):41 8-420. doi:10.1001/archneur.57.3.418

4. Skolnick P. Beyond monoamine-based therapies: clues to new approaches. J Clin Psychiatry. 2002;63:19-23.

5. Rana T, Behl T, Mehta V, et al. Molecular insights into the therapeutic promise of targeting HMGB1 in depression. Pharmacol Rep. 2021;73(1):31-42. doi:10.1007/s43440-020-00163-6

6. Lindqvist D, Dhabhar FS, James SJ, et al. Oxidative stress, inflammation and treatment response in major depression. Psychoneuroendocrinology. 2017;76:197-205. doi:10.1016/j.psyneuen.2016.11.031

7. Schiepers OJ, Wichers MC, Maes M. Cytokines and major depression. Prog Neuropsychopharmacol Biol Psychiatry. 2005;29 (2):201-217. doi:10.1016/j.pnpbp.2004.11.003

8. Shaftel SS, Carlson TJ, Olschowka JA, Kyrkanides S, Matousek SB, O'Banion MK. Chronic interleukin-1 $\beta$ expression in mouse brain leads to leukocyte infiltration and neutrophil-independent bloodbrain barrier permeability without overt neurodegeneration. $J$ Neurosci. 2007;27(35):9301-9309.

9. DiSabato DJ, Quan N, Godbout JP. Neuroinflammation: the devil is in the details. J Neurochem. 2016;Suppl 139(Supp12):136-153. doi:10.1111/jnc. 13607

10. Boche D, Perry VH, Nicoll JA. Review: activation patterns of microglia and their identification in the human brain. Neuropathol Appl Neurobiol. 2013;39(1):3-18. doi:10.1111/nan.12011

11. Priscila GCA, Joao VN, Jean PO, Elisa B, Mirian AFH. Neuroinflammation and glial cell activation in mental disorders. Brain Behav Immun. 2020;2:100034.

12. Yang TY, Jang EY, Ryu Y, et al. Effect of acupuncture on lipopolysaccharide-induced anxiety-like behavioural changes: involvement of serotonin system in dorsal Raphe nucleus. BMC Complement Altern Med. 2017;17(1):528-536. doi:10.1186/s12906-017-2039-y

13. Sriram CS, Jangra A, Gurjar SS, et al. Poly (ADP-ribose) polymerase-1 inhibitor, 3-aminobenzamide pretreatment ameliorates lipopolysaccharide induced neurobehavioral and neurochemical anomalies in mice. Pharmacol Biochem Behav. 2015;133:83-91. doi:10.1016/j.pbb.2015.03.022

14. Jangra A, Kwatra M, Singh T, et al. Piperine augments the protective effect of curcumin against lipopolysaccharide-induced neurobehavioral and neurochemical deficits in mice. Inflammation. 2016;39 (3):1025-1038. doi:10.1007/s10753-016-0332-4

15. O'Connor JC, Lawson MA, André C, et al. Lipopolysaccharide induced depressive-like behavior is mediated by indoleamine 2,3-dioxygenase activation in mice. Mol Psychiatry. 2009;14 (5):511-522. doi:10.1038/sj.mp.4002148

16. Jangra A, Lukhi MM, Sulakhiya K, Baruah CC, Lahkar M. Protective effect of mangiferin against lipopolysaccharide-induced depressive and anxiety-like behaviour in mice. Eur J Pharmacol. 2014; 740:337-345. doi:10.1016/j.ejphar.2014.07.031 
17. Huang Y, Henr CJ, Dantzer R, Johnson RW, Godbout JP. Exaggerated sickness behavior and brain proinflammatory cytokine expression in aged mice in response to intracerebroventricular lipopolysaccharide. Neurobiol Aging. 2008;29(11):1744-1753. doi:10.1016/j.neurobiolaging.2007.04. 012

18. Wardyn JD, Ponsford AH, Sanderson CM. Dissecting molecular cross-talk between Nrf2 and NF- $\mathrm{KB}$ response pathways. Biochem Soc Trans. 2015;43(4):621-626. doi:10.1042/BST20150014

19. Ahmed SM, Luo L, Namani A, Wang XJ, Tang X. Nrf2 signaling pathway: pivotal roles in inflammation. Biochim Biophys Acta Mol Basis Dis. 2017;1863(2):585-597. doi:10.1016/j.bbadis.2016.11.005

20. Lee JM, Johnson JA. An important role of Nrf2-ARE pathway in the cellular defense mechanism. J Biochem Mol Biol. 2004;37 (2):139-143. doi:10.5483/bmbrep.2004.37.2.139

21. Mohsin Alvi A, Tariq Al Kury L, Umar IM, et al. Post-treatment of synthetic polyphenolic 1,3,4 oxadiazole compound A3, attenuated ischemic stroke-induced neuroinflammation and neurodegeneration. Biomolecules. 2020;10(6):816. doi:10.3390/biom10060816

22. Kensler TW, Wakabayashi N, Biswal S. Cell survival responses to environmental stresses via the Keap1-Nrf2-ARE pathway. Annu Rev Pharmacol Toxicol. 2007;47(1):89-116. doi:10.1146/annurev. pharmtox.46.120604.141046

23. Cuadrado A, Manda G, Hassan A, et al. Transcription factor NRF2 as a therapeutic target for chronic diseases: a systems medicine approach. Pharmacol Rev. 2018;70(2):348-383. doi:10.1124/pr.11 7.014753

24. Uddin MS, Mamun AA, Jakaria M, et al. Emerging promise of sulforaphane-mediated Nrf2 signaling cascade against neurological disorders. Sci Total Environ. 2020;707:135624. doi:10.1016/j. scitotenv.2019.135624

25. Malik I, Shah FA, Ali T, et al. Potent natural antioxidant carveol attenuates MCAO-stress induced oxidative, neurodegeneration by regulating the Nrf-2 pathway. Front Neurosci. 2020;14:659. doi:10. 3389/fnins.2020.00659

26. Johnson DA, Johnson JA. Nrf2-a therapeutic target for the treatment of neurodegenerative diseases. Free Radic Biol Med. 2015;88(Pt B):253-267. doi:10.1016/j.freeradbiomed.2015.07.147

27. Zhu Y, Carvey PM, Ling Z. Altered glutathione homeostasis in animals prenatally exposed to lipopolysaccharide. Neurochem Int. 2007;50(4):671-680. doi:10.1016/j.neuint.2006.12.013

28. Martín-de-saavedra MD, Budni J, Cunha MP, et al. Nrf2 participates in depressive disorders through an anti-inflammatory mechanism. Psychoneuroendocrinology. 2013;38(10):2010-2022. doi:10.1016/j. psyneuen.2013.03.020

29. Uddin MS, Kabir MT, Mamun AA, et al. Pharmacological approaches to mitigate neuroinflammation in Alzheimer's disease. Int Immunopharmacol. 2020;84:106479.

30. Nagoor MMF, Javed H, Al TH, Azimullah S, Ojha SK. Pharmacological properties and molecular mechanisms of Thymol: prospects for its therapeutic potential and pharmaceutical development. Front Pharmacol. 2017;8:380.

31. Yu H, Zhang ZL, Chen J, et al. Carvacrol, a food-additive, provides neuroprotection on focal cerebral ischemia/reperfusion injury in mice. PLoS One. 2012;7(3):e33584. doi:10.1371/journal.pone.0033584

32. Lee B, Yeom M, Shim I, Lee H, Hahm DH. Inhibitory effect of carvacrol on lipopolysaccharide-induced memory impairment in rats. Korean J Physiol Pharmacol. 2020;24(1):27-37. doi:10.4196/kjpp. 2020.24.1.27

33. Melo FH, Moura BA, de Sousa DP, et al. Antidepressant-like effect of carvacrol (5-Isopropyl-2-methylphenol) in mice: involvement of dopaminergic system. Fundam Clin Pharmacol. 2011;25(3):362-367. doi:10.1111/j.1472-8206.2010.00850.x

34. Wang P, Luo Q, Qiao H, et al. The neuroprotective effects of carvacrol on ethanol-induced hippocampal neurons impairment via the antioxidative and antiapoptotic pathways. Oxid Med Cell Longev. 2017;2017:4079425.
35. Ventorp F, Bay-Richter C, Nagendra AS, et al. Exendin-4 treatment improves LPS-induced depressive-like behavior without affecting pro-inflammatory cytokines. J Parkinsons Dis. 2017;7(2):263-273. doi: $10.3233 /$ JPD-171068

36. Machado DG, Cunha MP, Neis VB, et al. Fluoxetine reverses depressive-like behaviors and increases hippocampal acetylcholinesterase activity induced by olfactory bulbectomy. Pharmacol Biochem Behav. 2012;103(2):220-229. doi:10.1016/j.pbb.2012.08.024

37. Jiang P, Guo Y, Dang R, et al. Salvianolic acid B protects against lipopolysaccharide-induced behavioral deficits and neuroinflammatory response: involvement of autophagy and NLRP3 inflammasome. J Neuroinflammation. 2017;14(1):239. doi:10.1186/s12974-017-1013-4

38. Choubey P, Kwatra M, Pandey SN, et al. Ameliorative effect of fisetin against lipopolysaccharide and restraint stress-induced behavioral deficits via modulation of NF- $\mathrm{kB}$ and IDO-1. Psychopharmacology (Berl). 2019;236(2):741-752. doi:10.1007/s00213-018-5105-3

39. Imran M, Shah FA, Nadeem H, et al. Synthesis and biological evaluation of benzimidazole derivatives as potential neuroprotective agents in an ethanol-induced rodent model. ACS Chem Neurosci. 2021;12(3):489-505. doi:10.1021/acschemneuro.0c00659

40. Iqbal S, Shah FA, Naeem K, et al. Succinamide derivatives ameliorate neuroinflammation and oxidative stress in scopolamine-induced neurodegeneration. Biomolecules. 2020;10(3):443. doi:10.3390/ biom 10030443

41. Rahman Z, Al Kury LT, Alattar A, et al. Carveol a naturally-derived potent and emerging nrf2 activator protects against acetaminophen-induced hepatotoxicity. Front Pharmacol. 2021;11:2448. doi:10.3389/fphar. 2020.621538

42. Ali A, Shah FA, Zeb A, et al. NF-kB inhibitors attenuate MCAO induced neurodegeneration and oxidative stress-a reprofiling approach. Front Mol Neurosci. 2020;13:33. doi:10.3389/fnmol.2020. 00033

43. Al Kury LT, Dayyan F, Ali Shah F, et al. Ginkgo biloba extract protects against methotrexate-induced hepatotoxicity: a computational and pharmacological approach. Molecules. 2020;25(11):2540. doi:10.3390/ molecules 25112540

44. Ullah U, Badshah H, Malik Z, et al. Hepatoprotective effects of melatonin and celecoxib against ethanol-induced hepatotoxicity in rats. Immunopharmacol Immunotoxicol. 2020;42(3):255-263. doi:10. 1080/08923973.2020.1746802

45. Ling L, Alattar A, Tan Z, et al. A potent antioxidant endogenous neurohormone melatonin, rescued MCAO by attenuating oxidative stress-associated neuroinflammation. Front Pharmacol. 2020;11: 1220. doi: $10.3389 /$ fphar.2020.01220

46. Shah FA, Gim SA, Kim MO, Koh PO. Proteomic identification of proteins differentially expressed in response to resveratrol treatment in middle cerebral artery occlusion stroke model. J Vet Med Sci. 2014;76(10):1367-1374. doi:10.1292/jvms.14-0169

47. Verdonk ML, Cole JC, Hartshorn MJ, Murray CW, Taylor RD. Improved protein-ligand docking using GOLD. Proteins. 2003;52 (4):609-623. doi:10.1002/prot.10465

48. Winkel AF, Engel CK, Margerie D, et al. Characterization of RA839, a noncovalent small molecule binder to Keap1 and selective activator of Nrf2 signaling. J Biol Chem. 2015;290(47):28446-28455. doi:10. 1074/jbc.M115.678136

49. Marcotte D, Zeng W, Hus JC, et al. Small molecules inhibit the interaction of Nrf2 and the Keap1 Kelch domain through a non-covalent mechanism. Bioorg Med Chem. 2013;21(14):40 11-4019. doi:10.1016/j.bmc.2013.04.019

50. Kumar GP, Khanum F. Neuroprotective potential of phytochemicals. Pharmacogn Rev. 2012;6(12):81-90. doi:10.4103/0973-7847.99898

51. Arigesavan K, Sudhandiran G. Carvacrol exhibits anti-oxidant and anti-inflammatory effects against 1, 2-dimethyl hydrazine plus dextran sodium sulfate induced inflammation associated carcinogenicity in the colon of Fischer 344 rats. Biochem Biophys Res Commun. 2015;461(2):314-320. doi:10.1016/j.bbrc.2015.04.030 
52. Lee S, Kim HB, Hwang ES, et al. Antidepressant-like effects of p-coumaric acid on LPS-induced depressive and inflammatory changes in Rats. Exp Neurobiol. 2018;27(3):189-199. doi:10.5607/ en.2018.27.3.189

53. Sulakhiya K, Keshavlal GP, Bezbaruah BB, et al. Lipopolysaccharide induced anxiety- and depressive-like behaviour in mice are prevented by chronic pre-treatment of esculetin. Neurosci Lett. 2016;611: 106-111. doi:10.1016/j.neulet.2015.11.031

54. Blanco MM, Costa CA, Freire AO, Santos JG Jr, Costa M. Neurobehavioral effect of essential oil of Cymbopogon citratus in mice. Phytomedicine. 2009;16(2-3):265-270. doi:10.1016/j.phymed. 2007.04.007

55. Liu Z, Zhou T, Ziegler AC, Dimitrion P, Zuo L. Oxidative stress in neurodegenerative diseases: from molecular mechanisms to clinical applications. Oxid Med Cell Longev. 2017;2017:2525967. doi:10.11 $55 / 2017 / 2525967$

56. Uddin MS, Al Mamun A, Kabir MT, et al. Neuroprotective role of polyphenols against oxidative stress-mediated neurodegeneration. Eur J Pharmacol. 2020;886:173412.

57. Thakare VN, Dhakane VD, Patel BM. Potential antidepressant-like activity of silymarin in the acute restraint stress in mice: modulation of corticosterone and oxidative stress response in cerebral cortex and hippocampus. Pharmacol Rep. 2016;68(5):1020-1027. doi:10.1016/j. pharep.2016.06.002

58. Salim S. Oxidative stress and the central nervous system. J Pharmacol Exp Ther. 2017;360(1):201-205. doi:10.1124/jpet.116.237503

59. Felicity N, Berk M, Dean O, Ashley I. Bush, oxidative stress in psychiatric disorders: evidence base and therapeutic implications. Int J Neuropsychopharmacol. 2008;11(6):851-876.

60. Thimmulappa RK, Scollick C, Traore K, et al. Nrf2-dependent protection from LPS induced inflammatory response and mortality by CDDO-Imidazolide. Biochem Biophys Res Commun. 2006;351 (4):883-889. doi:10.1016/j.bbrc.2006.10.102

61. Liao D, Chuanfeng L, Cao L, et al. Curcumin attenuates chronic unpredictable mild stress-induced depressive-like behaviors via restoring changes in oxidative stress and the activation of $\mathrm{Nrf} 2$ signaling pathway in rats. Oxid Med Cell Longev. 2020;2020.
62. Dean B, Tawadros N, Scarr E, Gibbons AS. Regionally-specific changes in levels of tumour necrosis factor in the dorsolateral prefrontal cortex obtained postmortem from subjects with major depressive disorder. J Affect Disord. 2010;120:245-248. doi:10.10 16/j.jad.2009.04.027

63. Dowlati Y, Herrmann N, Swardfager W, Liu H, Sham L, Reim EK. A meta-analysis of cytokines in major depression. Biol Psychiatry. 2010;67(5):446-457. doi:10.1016/j.biopsych.2009.09.033

64. Yao W, Zhang JW, Ishima T, et al. Role of Keap1-Nrf2 signaling in depression and dietary intake of glucoraphanin confers stress resilience in mice. Sci Rep. 2016;6(1):30659. doi:10.1038/srep30659

65. Kim BW, Koppula S, Hong SS, et al. Regulation of microglia activity by glaucocalyxin-A: attenuation of lipopolysaccharide-stimulated neuroinflammation through NF-kappaB and p38 MAPK signaling pathways. PLoS One. 2013;8(2):e55792. doi:10.1371/journal.pone. 0055792

66. Kim J, Cha YN, Surh YJ. A protective role of nuclear factor-erythroid 2-related factor-2 (Nrf2) in inflammatory disorders. Mutat Res. 2010;690(1-2):12-23. doi:10.1016/j.mrfmmm.2009.09. 007

67. Luo JF, Shen XY, Lio CK, et al. Activation of Nrf2/HO-1 pathway by nardochinoid $\mathrm{c}$ inhibits inflammation and oxidative stress in lipopolysaccharide-stimulated macrophages. Front Pharmacol. 2018;9:911. doi:10.3389/fphar.2018.00911

68. Zulfiqar Z, Shah FA, Shafique S, et al. Repurposing FDA Approved Drugs as JNK3 Inhibitor for Prevention of Neuroinflammation Induced by MCAO in Rats. J Inflamm Res. 2020;13:1185-1205. doi:10.2147/JIR.S284471

69. Sang Eun H, Seong Min K, Ho Jeong L, et al. Scutellarein Induces Fas-Mediated extrinsic apoptosis and G2/M cell cycle arrest in hep3b hepatocellular carcinoma cells. Nutrients. 2019;11(2):263. doi:10.33 90/nu11020263

70. Louneva N, Cohen JW, Han LY, et al. Caspase-3 is enriched in postsynaptic densities and increased in Alzheimer's disease. Am J Pathol. 2008;173(5):1488-1495. doi:10.2353/ajpath.2008.080434
Journal of Inflammation Research

\section{Publish your work in this journal}

The Journal of Inflammation Research is an international, peerreviewed open-access journal that welcomes laboratory and clinical findings on the molecular basis, cell biology and pharmacology of inflammation including original research, reviews, symposium reports, hypothesis formation and commentaries on: acute/chronic inflammation; mediators of inflammation; cellular processes; molecular mechanisms; pharmacology and novel anti-inflammatory drugs; clinical conditions involving inflammation. The manuscript management system is completely online and includes a very quick and fair peerreview system. Visit http://www.dovepress.com/testimonials.php to read real quotes from published authors. 